Article

\title{
Benchmark Dataset Based on Category Maps with Indoor-Outdoor Mixed Features for Positional Scene Recognition by a Mobile Robot ${ }^{\dagger}$
}

\author{
Hirokazu Madokoro $^{1, * \mathbb{C}}$, Hanwool Woo ${ }^{2}{ }^{\mathbb{C}}$, Stephanie Nix ${ }^{1}$ and Kazuhito Sato ${ }^{1}$ \\ 1 Faculty of Systems Science and Technology, Akita Prefectural University, Yurihonjo City 015-0055, Japan; \\ snix@akita-pu.ac.jp (S.N.); ksato@akita-pu.ac.jp (K.S.) \\ 2 Institute of Engineering Innovation, The University of Tokyo, Bunkyo-ku, Tokyo 113-8656, Japan; \\ woo@robot.t.u-tokyo.ac.jp \\ * Correspondence: madokoro@akita-pu.ac.jp; Tel.: +81-184-27-2180 \\ $+\quad$ This paper is an extended version of our paper published in Fukushi, R.; Madokoro, H.; Sato, K. Semantic \\ Scene Recognition and Zone Labeling for Mobile Robot Benchmark Datasets based on Category Maps. \\ In Proceedings of 18th International Conference on Control, Automation and Systems, Daegwallyeong, \\ Korea, 17-20 October 2018; pp. 475-480.
}

Received: 28 April 2020; Accepted: 25 May 2020; Published: 26 May 2020

\begin{abstract}
This study was conducted to develop original benchmark datasets that simultaneously include indoor-outdoor visual features. Indoor visual information related to images includes outdoor features to a degree that varies extremely by time, weather, and season. We obtained time-series scene images using a wide field of view (FOV) camera mounted on a mobile robot moving along a 392-m route in an indoor environment surrounded by transparent glass walls and windows for two directions in three seasons. For this study, we propose a unified method for extracting, characterizing, and recognizing visual landmarks that are robust to human occlusion in a real environment in which robots coexist with people. Using our method, we conducted an evaluation experiment to recognize scenes divided up to 64 zones with fixed intervals. The experimentally obtained results using the datasets revealed the performance and characteristics of meta-parameter optimization, mapping characteristics to category maps, and recognition accuracy. Moreover, we visualized similarities between scene images using category maps. We also identified cluster boundaries obtained from mapping weights.
\end{abstract}

Keywords: counter propagation network; human detection; mobile robot; part-based features; saliency maps; scene recognition; self-organizing maps; U-Matrix; visual landmarks

\section{Introduction}

With the rapid progress of recent artificial intelligence (AI) and robotic technologies [1], widely various intelligent robots [2] have been developed for industrial utilization at factories and warehouses. They have also been developed for collaborative utilization in human societies and facilities in terms of homes [3], offices [4], kindergartens [5], nursing-care facilities [6], and hospitals [7]. To perform autonomous locomotion, robots must have capabilities to perform accurate actions and functions of self-localization, path planning and tracking, object recognition, and environmental understanding [8]. Particularly for mutual dependence and coexistence in human society, robots must have functions to detect people and to recognize human actions and activities effectively [9].

Simultaneous localization and mapping (SLAM) technologies [10] have been studied widely as a fundamental approach for autonomous locomotion of mobile robots including drones [11]. For sequential processing and construction and update of a map for self-location estimation, 
the computational cost for SLAM increases because probability calculations are necessary for extraction of various map-creation features and for updating real-time positional information. Moreover, the storage capacity for creating environmental maps is expanded exponentially because of the increased observation areas according to enhanced robot locomotion. As an alternative approach, autonomous locomotion using landmarks has been specifically examined as a topic of computer and robot vision (RV) studies [12]. Advance creation of maps is unnecessary for landmark-based autonomous locomotion and navigation [13]. Therefore, this approach is effective for considerable reduction of storage capacity and computational costs. However, a difficulty arises: how to set up landmarks. Installing landmarks in advance is another difficulty inherent in this approach.

Landmarks with high discrimination accuracy installed in an environment beforehand according to the purpose and resolution can minimize the ability and performance of a robot in terms of their recognition capability. However, this approach not only involves a pre-installation burden and periodic maintenance, it also involves restrictions for locomotion only in a pre-installed environment. As an approach that requires no landmark pre-installation, visual landmarks (VLs) have been attracting attention in the past two decades [14]. Herein, VLs are defined as visually prominent objects including text and feature patterns in a scene [15]. Mohareri et al. [16] proposed a navigation method using augmented reality (AR) markers as landmarks. Although AR markers have high discriminative performance, their installation involves a great burden and various difficulties. Unlike AR markers, we consider that VLs extracted from general objects have high affinity from the viewpoint of semantic recognition among people because of the huge amounts of environmental information from vision. However, a challenging task of robot-vision studies is to extract accurate and suitable VLs that have both robustness and stability under environmental changes every moment.

As a pioneering study, Hayet et al. [12] proposed a VL framework for indoor mobile robot navigation. They extracted quadrangular surfaces as VL candidates from scene images based on horizontal and vertically oriented edges. Finally, VLs are extracted from doors and posters using random sample consensus (RANSAC) [17]. The experimentally obtained results revealed that the recognition accuracy achieved $80 \%$ for their original navigation benchmark datasets. Moreover, they obtained $90 \%$ and greater accuracy for a wider environment. However, they considered no environmental changes in their benchmark datasets.

Numerous datasets have been proposed for the classification, recognition, and understanding of scenes and objects based on visual information [18-23]. These datasets comprise learning, validation, and testing subsets for evaluating generalization capability in various and diverse environmental changes. Recently, three-dimensional (3D) datasets obtained using drone-mounted cameras, especially for multiple object tracking (MOT) tasks [24], have been expanding [25]. Therefore, scene recognition has been extended from a two-dimensional (2D) plane to a 3D space [26]. In outdoor environments, global navigation satellite system (GNSS) signals are used for precision position estimation combined with vision sensors [27].

The numerous and diverse benchmark datasets developed for various purposes can be classified roughly into two types: indoor scene datasets [28] and outdoor scene datasets [29]. Nevertheless, no dataset simultaneously including indoor-outdoor visual features has been proposed. Scene recognition is evaluated separately for indoor datasets and outdoor datasets [30]. Realistically, outdoor scene features are included in indoor scenes because transparent glass walls are used occasionally for lighting and artistic building design. The occasions of indoor scene features including outdoor areas are few.

Visual information in indoor images including outdoor features varies considerably in terms of time, weather, and season. Figure 1 depicts daytime and nighttime scene images in the same position. A great difference arises because of effects from the outside through a transparent glass window. Scene recognition studies using a mobile robot are used in a few cases for accuracy comparison in visual information changes at similar positions. The partial occlusion, corruption, and distortion caused by a limited field of view (FOV) of a camera occur frequently everywhere, 
depending not only on environmental changes but also on locomotion paths with avoidance of static and dynamic obstacles [31]. Evaluation criteria and benchmark datasets for these requirements are under development.
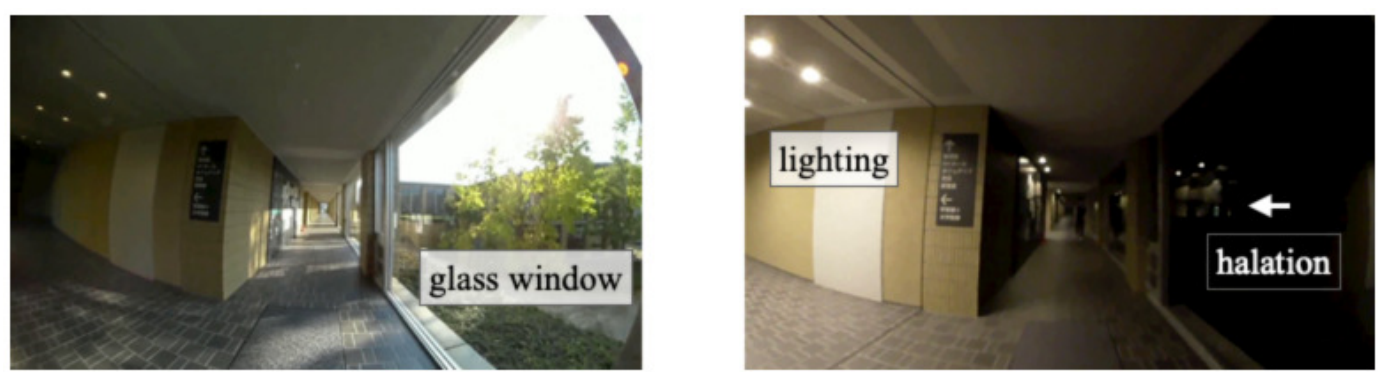

Figure 1. Difference of similar scenes in daytime and nighttime at the same position.

For our earlier study [32], we proposed a unified method for extracting, characterizing, and recognizing VLs that were robust to human occlusion in a real environment in which robots coexist with people. Based on classically established machine-learning (ML) technologies, our earlier method [32] comprised the following five procedures: VL extraction from generic objects using a saliency map (SM) [33]; part-based feature description using accelerated KAZE (AKAZE) [34]; human region extraction using histograms of oriented gradients (HOGs) [35]; codebook creation using self-organizing maps (SOMs) [36]; and positional scene recognition using counter propagation networks (CPNs) [37]. The contributions of this study are the following.

1. We strove to evaluate the robustness of our improved semantic scene recognition method for extending environmental changes using novel datasets.

2. We develop our original scene recognition benchmark datasets in which indoor-outdoor visual features coexist.

3. We evaluate the effects of outdoor features for indoor scene recognition for weather and seasonal changes outdoors.

4. We evaluate details and quantitative evaluation for visualizing classification results obtained using category maps.

The rest of the paper is structured as follows. In Section 2, we present our proposed method based on computer vision and machine-learning algorithms of several types. Section 3 presents our original benchmark datasets of time-series scene images obtained using a wide FOV camera mounted on a mobile robot in an indoor environment surrounded by transparent glass walls and windows for two directions in three seasons. Subsequently, Section 4 presents evaluation experimentally obtained results of the performance and characteristics of meta-parameter optimization, mapping characteristics to category maps, and recognition accuracy. Finally, Section 5 concludes and highlights future work. Herein, we had proposed this basic method in the proceeding [38] (C)2018 IEEE. For this paper, we have described detailed results using our novel benchmark datasets.

\section{Proposed Method}

Our proposed method comprises two modules: a feature-extraction module and a scene recognition module. Figures 2 and 3 depict the flow processing and structures of the respective modules. The feature-extraction module performs saliency-based VL extraction and feature description from input images. This module comprises three algorithms: You Only Look Once (YOLO) [39], SMs [33], and AKAZE [34]. First, using YOLO, human regions are extracted from a scene image. Although HOG [35] was used for our earlier study [32], the human detection accuracy with HOG, which includes insufficient robustness to rotation and scaling, was increased by only $60 \%$. For this study, we extract bounding boxes (BBs) that include pedestrians using YOLO. We remove feature points inside 
BBs from VL candidates. Subsequently, salient regions are extracted using SMs [33]. Finally, features are described using AKAZE from highly salient regions as VLs. Although the feature-extraction module from our earlier study is reused [32], we introduce YOLO for the novelty of this method.

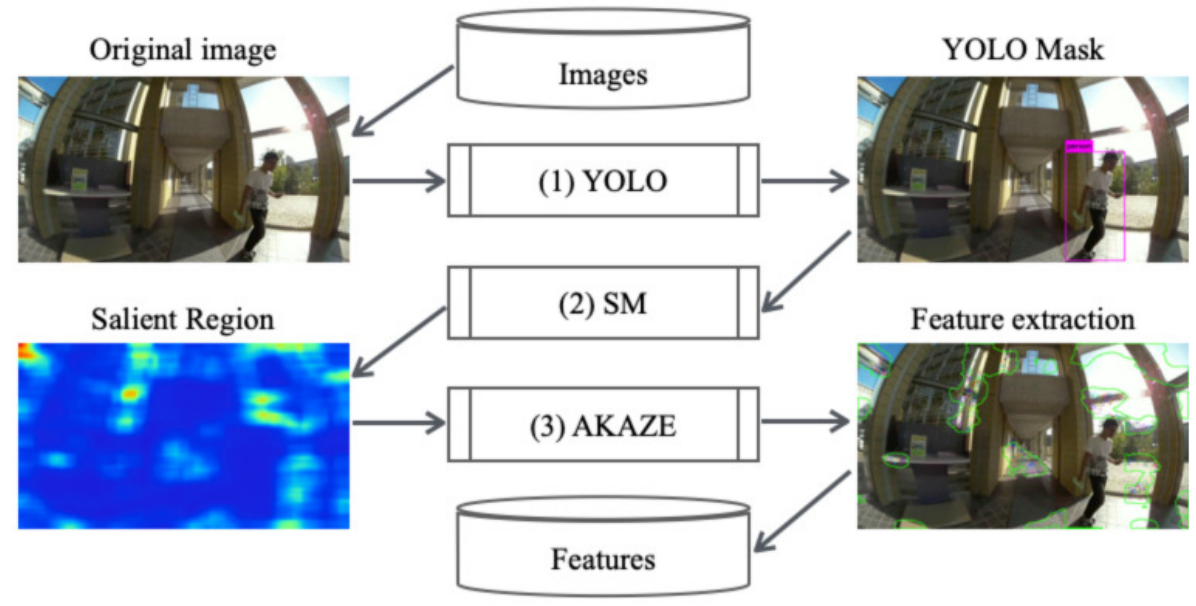

Figure 2. Proposed method of the feature-extraction module for visual landmark (VL) extraction and part-based feature description.

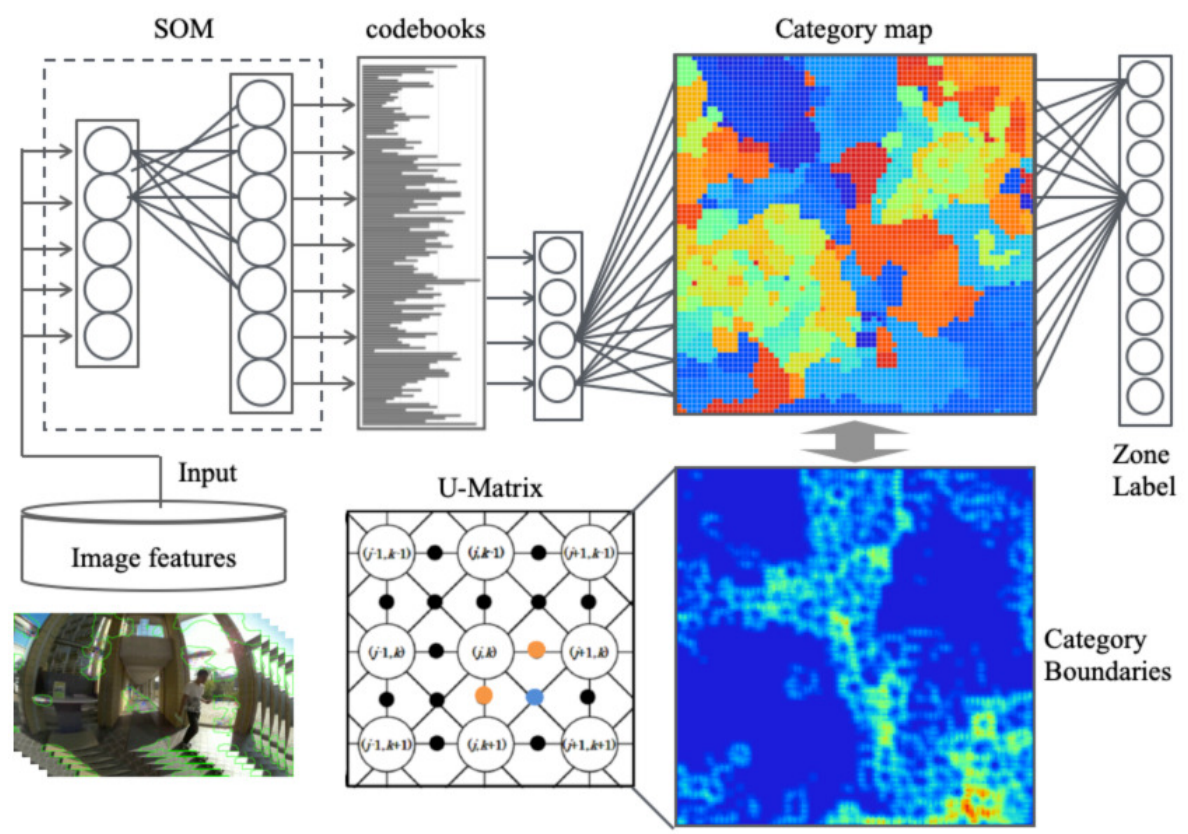

Figure 3. Proposed method of the recognition module for scene recognition and cluster boundary extraction of category map.

Thereafter, positions are recognized as zones from scene images with the recognition module, as depicted in Figure 3. The recognition module comprises three algorithms: SOMs [36], CPNs [37], and U-Matrix [40]. Input features are compressed with SOMs for a unified dimension as codebooks. The CPN learning phase performs category map generation and visualization of the similarity of codebooks. The CPN verification phase recognizes scene images as zones using category maps for verification scene images. Moreover, with reference to another earlier method [41], we use U-Matrix [40] to extract cluster boundaries from weights on category maps. Cluster boundaries are enhanced 
for differences between labels and weights. Features and properties of these five algorithms are explained below.

\subsection{VL Extraction Using SM}

Human stereo vision [42] provides numerous and diverse real-world information [43]. Although various decisions are made based on visual information, the speed of processing visual information is rather slow because the amounts of information are large compared to those provided by tactile, auditory, and olfactory senses. Therefore, we have limitations on the use of all visual information for recognition and decision-making. In contrast, we have a mechanism to devote attention solely to some things that should be noticed. Actually, SMs were developed as a mechanism for finding objects that must command attention.

After appending return suppression in similar frames, as a novel application for this study, we extend SMs, which are based on processing for a single image, to time-series images. In addition, recovery was strengthened between consecutive frames for steady VL detection. The brief procedures of saliency maps include the following five steps. First, a pyramid image is created from codebooks for use as input data. Second, a Gaussian filter is applied to the pyramid image. Third, images of the respective components of color phase, brightness, and direction are created. Fourth, feature maps (FMs) are created as visual features of the respective components with center-surround and normalization operations. Finally, saliency maps $I_{s m}$ are obtained from a winner-take-all (WTA) competition for the linear summation of FMs.

\subsection{Feature Description Using AKAZE}

For conventional generic object recognition, scale-invariant feature transform (SIFT) [44], SURF [45], binary robust independent elementary features (BRIEF) [46], and oriented features from accelerated segment test (FAST) and rotated BRIEF (ORB) [47] descriptors have been used widely as outstanding descriptors of local features. Table 1 summerizes feature dimensions in respective descriptors. Actually, SIFT descriptors are robust for rotation, scale, position, and brightness changes, not only from static images but also from dynamic images. Alcantarilla et al. [48] proposed KAZE using nonlinear scale-space filtering as a feature intended to exceed the SIFT performance. Moreover, they proposed AKAZE [34], which accelerated the KAZE construction. In contrast to SIFT, AKAZE was demonstrated as being approximately three times faster, although maintaining equivalent performance and accuracy. Therefore, we use AKAZE, which is suitable for indoor environments where environmental changes occurred frequently.

Table 1. Feature dimensions of representative descriptors [dimensions].

\begin{tabular}{cccccc}
\hline SIFT & SURF & ORB & BRIEF & KAZE & AKAZE \\
\hline 128 & $128 / 64$ & 32 & $64 / 32 / 16$ & 128 & 61 \\
\hline
\end{tabular}

To achieve acceleration of feature detection, AKAZE employs fast explicit diffusion (FED) [49], which is embedded in a pyramidal framework in nonlinear scale-space filters. Comparison with additive operator splitting [50] schemes used in their former descriptor KAZE [48] shows that FED schemes are more accurate. They also provide extremely easy implementation. Moreover, AKAZE introduced a highly efficient Modified-Local Difference Binary descriptor [51] to preserve low computational demand and storage requirements. The numbers of features points differ in the respective images. Letting $I_{a k a z e}$ be a set of AKAZE features points, then each feature point includes 64-dimensional feature vectors. 


\subsection{Human Detection Using YOLO}

Based on deep learning (DL) mechanisms [52], YOLO was proposed by Redmon et al. [39] as a real-time algorithm for object detection and class recognition. For performing both functions, YOLO is used widely for general object detection of various applications [53] such as pedestrians [54], cars [55], license plates [56], and accidents [57]. Moreover, YOLO performs high recognition accuracy and rapid processing speed compared with the single-shot multi-box detector [58]. Therefore, we used YOLO for human detection instead of HOG [35], which was used in our earlier study [32]. However, to keep accuracy in different shapes of human figures in terms of stand up, sitting, lying, and occluded body parts is important. We consider that this is a challenging task for our method combined with YOLO and OpenPose [59], which is an approach to efficiently detect the 2D pose of multiple people in an image.

The procedures necessary for YOLO are the following. First, the original image is resized into a square. Then the object is detected using a BB. Subsequently, divided grids perform object class and $\mathrm{BB}$ extraction in parallel for each region. Finally, the integration result in $I_{\text {yolo }}$ of both realizes another class of object recognition.

YOLO learns the surrounding context because the entire image is targeted for learning. This mechanism suppresses false detection in background areas. However, divided grids are limited to a fixed size. Moreover, YOLO has a restriction by which the detected objects in each grid can be as many as two because only a single class can be used for identification in each grid. As a shortcoming, the small objects included in each grid degrade the detection accuracy. Generic object detection is beyond the scope of this study. We intend to assess the accuracy of positional scene recognition from scene images with occlusion or deficiency.

\subsection{Codebook Description Using SOM}

We use SOMs [36] to create codebooks from AKAZE features. Let $x_{p}(t)$ represent the output from the input layer unit $p(1 \leq p \leq P)$ at time $t$. As input features, $I_{v l}$ are appended to $x_{p}(t)$ as VL features.

$$
I_{v l}=I_{s m} \wedge I_{a k a z e} \wedge I_{\text {yolo }} .
$$

Let $w_{p, q}(t)$ be a weight from $p$ to mapping layer unit $q(1 \leq q \leq Q)$ at time $t$. Herein, $P$ and $Q$ respectively denote the total numbers of input layer units and mapping layer units. Before learning, $w_{p, q}(t)$ are initialized randomly. Using the Euclidean distance between $x_{p}(t)$ and $w_{p, q}(t)$, a winner unit $c_{q}(t)$ is sought for the following.

$$
c_{q}(t)=\underset{1 \leq q \leq Q}{\operatorname{argmin}} \sqrt{\sum_{p=1}^{P}\left(x_{p}(t)-w_{p, q}(t)\right)^{2}} .
$$

A neighborhood region $\psi_{\text {som }}(t)$ is set from the center of $c_{q}$ as

$$
\psi_{\text {som }}(t)=\left\lfloor\psi_{\text {som }}(0) \cdot Q \cdot\left(1-\frac{t}{Z_{\text {som }}}\right)+0.5\right\rfloor,
$$

where $Z_{\text {som }}$ represents the maximum of learning iterations. Subsequently, $w_{p, q}(t)$ in $\psi_{\text {som }}(t)$ is updated as

$$
w_{p, q}(t+1)=w_{p, q}(t)+\alpha(t)\left(x_{p}(t)-w_{p, q}(t)\right),
$$

where $\alpha(t)$ is a learning coefficient that decreases according to the learning progress. Herein, at time $t=0$, we initialized $w_{p, q}(0)$ with random numbers. After WTA learning, test data are entered into the input layer. A winner unit is used for voting to create a histogram as a codebook: $I_{\text {som }}$. 


\subsection{Scene Recognition Using CPN}

We apply CPNs [37] to category maps from codebooks. For learning CPNs, $I_{\text {som }}$ are entered as input features to the input layer of CPNs. Let $y_{r}(t)$ be output from the input layer unit $r(1 \leq r \leq R)$ at time $t$. Let $u_{r, s}(t)$ be a weight from $r$ to Kohonen layer unit $s(1 \leq s \leq S)$ at time $t$. Moreover, let $v_{s, k}(t)$ be a weight from Grossberg layer unit $k$ to Kohonen layer unit $s(1 \leq s \leq S)$ at time $t$. Herein, $R$ and $Q$ respectively denote the total numbers of input layer units and Kohonen layer units. Before learning, $w_{r, s}(t)$ are initialized randomly. Using the Euclidean distance between $y_{r}(t)$ and $u_{r, s}(t)$, a winner unit $c_{s}(t)$ is sought for the following.

$$
c_{s}(t)=\underset{1 \leq s \leq S}{\operatorname{argmin}} \sqrt{\sum_{r=1}^{R}\left(y_{r}(t)-u_{r, s}(t)\right)^{2}} .
$$

A neighborhood region $\psi_{c p n}(t)$ is set as the following from the center of $c_{s}$.

$$
\psi_{c p n}(t)=\left\lfloor\psi_{c p n}(0) \cdot S \cdot\left(1-\frac{t}{Z_{c p n}}\right)+0.5\right\rfloor
$$

In that equation, $Z_{c p n}$ stands for the maximum learning iteration. Subsequently, $u_{r, s}$ and $v_{s, k}$ in $\psi_{c p n}(t)$ are updated as

$$
\begin{aligned}
& u_{r, s}(t+1)=u_{r, s}(t)+\beta(t)\left(y_{r}(t)-u_{r, s}(t)\right), \\
& v_{s, k}(t+1)=v_{s, k}(t)+\gamma(t)\left(z_{l}(t)-v_{s, k}^{j}(t)\right),
\end{aligned}
$$

where $\beta(t)$ and $\gamma(t)$ are learning coefficients, which decrease along with learning progress. Herein, at time $t=0$, we initialized $u_{r, s}(0)$ and $v_{s, k}(0)$ with random numbers.

As a learning result, $u_{r, s}$ is used for the input to CNNs. We define this interface as $I_{c p n}$.

\subsection{Boundary Extraction Using U-Matrix}

We use U-Matrix [40] to extract boundaries from category maps. Let $s x$ and $s y$ be a unit index on a 2D category map. Cluster boundaries are extracted from $u_{r, s x, s y}$ using U-Matrix. Based on metric distances between weights, U-Matrix visualizes the spatial distribution of categories from the similarity of neighbor units [40]. On a 2D category map of square grids, a unit has eight neighbor units except for boundary units. Assuming $U$ as the similarity calculated using U-Matrix, then for the component of the horizontal and vertical directions, $U_{h \pm}$ and $U_{v \pm}$ are defined as shown below.

$$
\begin{aligned}
& U_{h \pm}=\sqrt{\sum_{r=1}^{R}\left(u_{r, s x, s y}-u_{r, s x \pm 1, s y}\right)^{2}}, \\
& U_{v \pm}=\sqrt{\sum_{r=1}^{R}\left(u_{r, s x, s y}-u_{r, s x, s y \pm 1}\right)^{2}} .
\end{aligned}
$$

For components of the diagonal directions, $U_{d \pm}$ are defined as the following.

$$
U_{d \pm}=\frac{1}{2} \sqrt{\sum_{r=1}^{R}\left(u_{r, s x, s y \pm 1}-u_{r, s x \pm 1, s y}\right)^{2}}+\frac{1}{2} \sqrt{\sum_{r=1}^{R}\left(u_{r, s x \pm 1, s y}-u_{r, s x, s y \pm 1}\right)^{2}}
$$




\section{Scene Recognition Benchmark Datasets}

We obtained original benchmark datasets to evaluate the fundamental performance, usefulness, and practicality of our proposed method using a mobile robot equipped with a camera. The primary characteristic of this dataset is that a long corridor inside of our university buildings surrounded by numerous transparent glass walls and windows affects outdoor features. For this study, we obtained scene images in three outdoor conditions: daytime in summer, nighttime in autumn, and daytime in winter.

\subsection{Mobile Robot and Camera}

We obtained video sequences using a fisheye lens camera mounted on a mobile robot. We used a two-wheeled inverted-pendulum mobile robot (Double; Double Robotics, Inc. Burlingame, CA, USA). The photograph on the right side of Figure 4 presents the robot appearance. The robot is $119 \mathrm{~cm}$ high with its neck moving up and down to a 31-cm span. For this experiment, we fixed the lowest neck position to maintain constant FOV and locomotion stability.

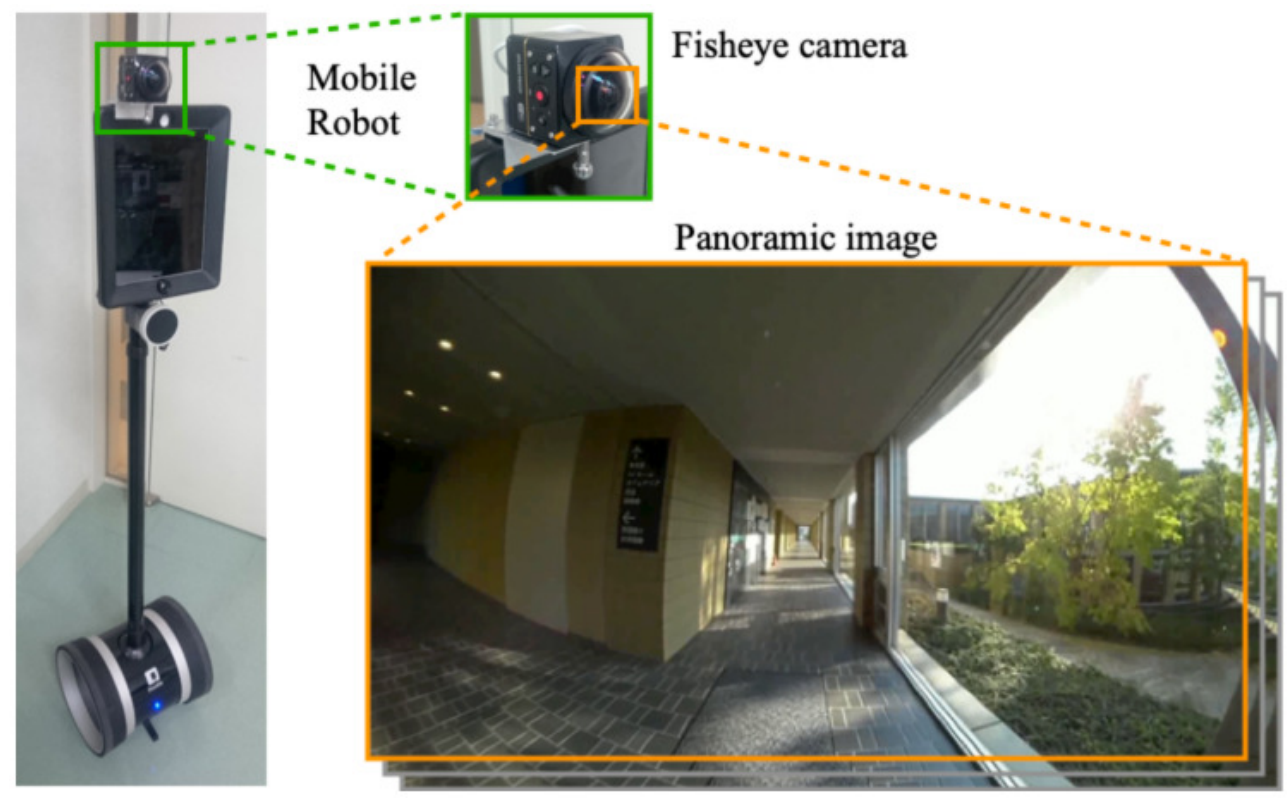

Figure 4. Mobile robot (Double; Double Robotics, Inc. Burlingame, CA, USA).), camera (PIXPRO SP360; Eastman Kodak Co. Rochester, NY, USA).), and a scene image in an environment where indoor-outdoor features are mixed.

For our earlier study [32], we obtained video sequences using a built-in camera of a tablet computer (iPad; Apple Inc. Cupertino, CA, USA).) mounted on the robot head part. The image resolution of $640 \times 480$ pixels was insufficient to capture objects as VLs. Moreover, the captured video sequences were sent to a laptop using a low-power wireless communication protocol because of a lack of a storage function in the tablet. For this experiment, we used a fisheye lens camera (PIXPRO SP360; Eastman Kodak Co. Rochester, NY, USA).) with high-resolution and wide-range FOV.

Table 2 shows major camera specifications. For this camera, the focal length of the lense is $0.805 \mathrm{~mm}$, which is equivalent to a length of $8.25 \mathrm{~mm}$ for a $35 \mathrm{~mm}$ film. This camera is fundamentally used with a vertical upward arrangement for the lens. We originally developed a camera mount using an L-shaped aluminum plate. Using this mount, we installed the camera at the front of the robot, as depicted in Figure 4. To ensure good resolution, we used the FOV of the 235-deg mode instead of the 360-deg mode. 
Table 2. Major specifications of the camera (PIXPRO SP360; Eastman Kodak Co.

\begin{tabular}{cc}
\hline Parameters & Values \\
\hline Imaging device & $1 / 2.33 \mathrm{CMOS}$ \\
FOV & $235 \mathrm{degs}$ \\
Resolution & $1280 \times 720$ pixels \\
Frame rate & $60 \mathrm{fps}$ \\
Focal length & $0.805 \mathrm{~mm}(8.20 \mathrm{~mm}$ for $35 \mathrm{~mm}$ film equivalent $)$ \\
Battery & $3.6 \mathrm{~V} ; 1250 \mathrm{mAh}$; Li-ion \\
Size & $48.0 \times 50.0 \times 52.5 \mathrm{~mm}$ \\
Weight & $103 \mathrm{~g}$ \\
\hline
\end{tabular}

\subsection{Experimental Environment}

Figure 5 presents a photograph and a map of the buildings at Honjo Campus $\left(40^{\circ} \mathrm{N}, 140^{\circ} \mathrm{E}\right)$, Akita Prefectural University, Akita, Japan. This campus is located in the countryside. There are three buildings: a lecture building, a common building, and a faculty building. Each building is connected by a crossing corridor. The approximate size of all buildings is $90 \mathrm{~m}$ measured longitudinally and $80 \mathrm{~m}$ measured laterally.
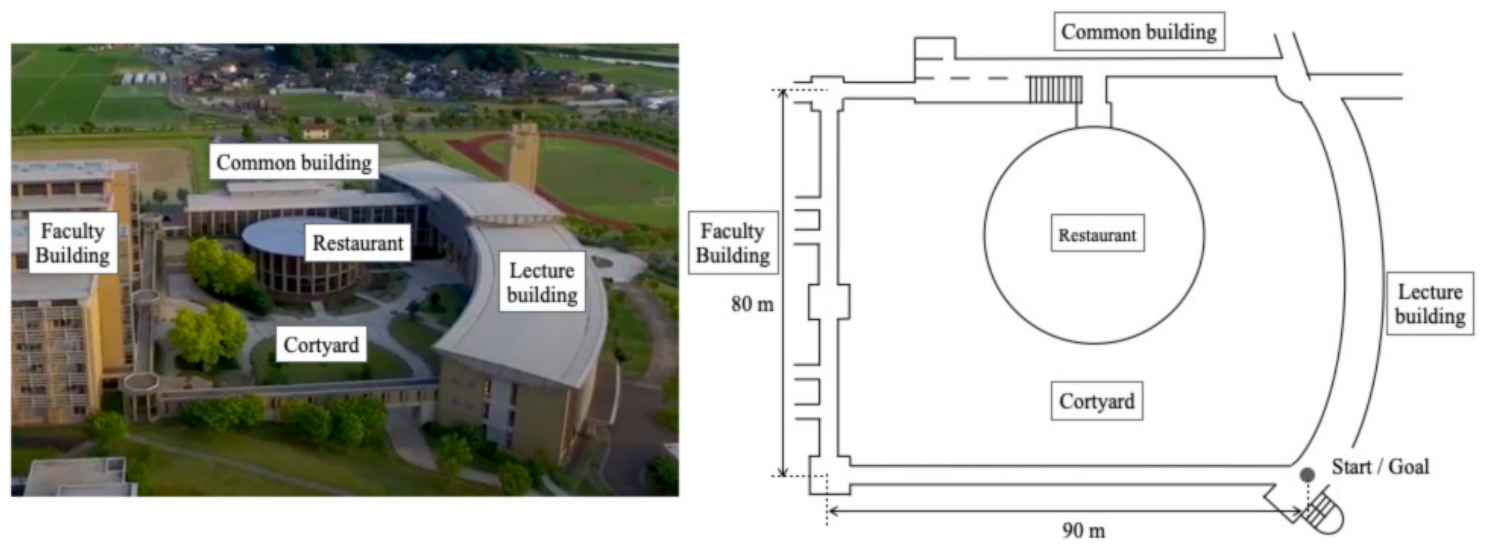

Figure 5. Map of the experiment environment.

The total locomotion distance of the robot is $392 \mathrm{~m}$ per round. The robot was operated manually using a keyboard on a laptop computer. An operator practiced adequately in advance. The robot moved at a constant speed when obtaining all datasets with no meandering locomotion.

Initially, we divided the route into four zones based on the four right-angle corners, as depicted in Figure 6a. Subsequently, the respective zones were divided into 2, 4, 8, and 16 refined zones of equal length. As depicted in Figure 6, 4, 8, 16, 32, and 64 zones were defined for evaluation granularity. We assigned ground truth (GT) labels for five patterns: Zones 1-4, Zones 1-8, Zones 1-16, Zones 1-32, and Zones 1-64.

We obtained video sequences in two directions: clockwise (CW) and counter-clockwise (CCW). The robot moved along the route three rounds in each direction. We obtained six video sequences for one outside condition. Moreover, we conducted experiments to obtain video sequences in three seasons: summer, autumn, and winter. The outside conditions differ among seasons: a summer sunny day in August, a cloudy autumn moonless night in October, and a snowy winter day in December. These weather conditions are typical of this region of northern Japan. For this study, we designated the respective datasets as summer datasets (SD), autumn datasets (AD), and winter datasets (WD). 


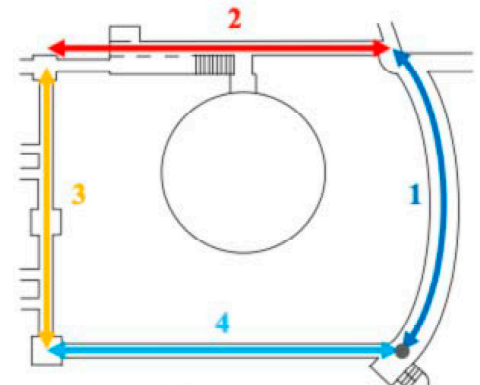

(a) 4 zones

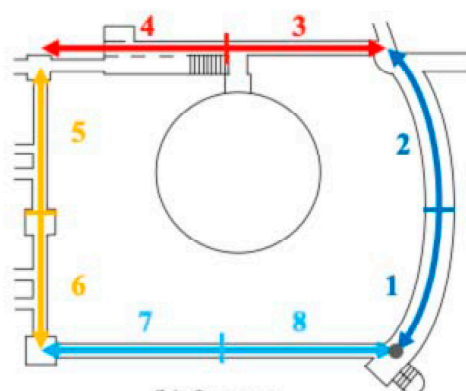

(b) 8 zones

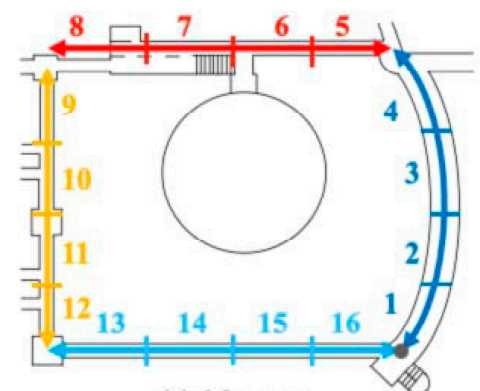

(c) 16 zones

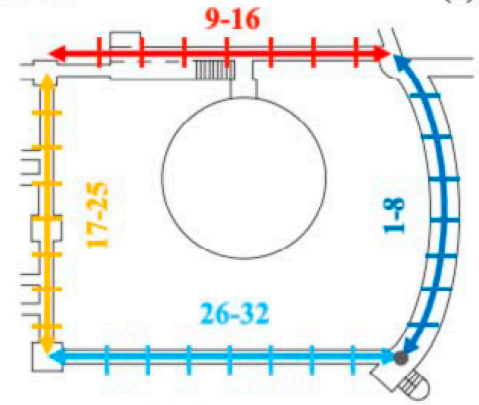

(d) 32 zones

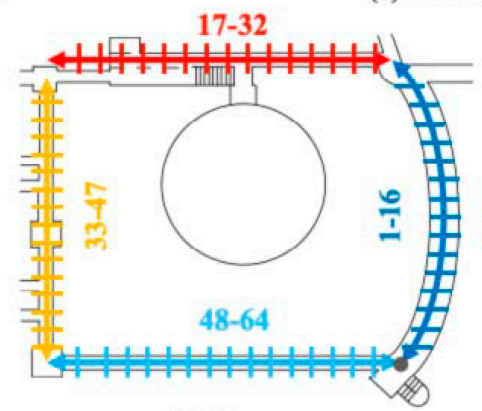

(e) 64 zones

Figure 6. Zones of five division types with fixed intervals.

\subsection{Obtained Indoor-Outdoor Mixed Scene Images}

For this study, we obtained video sequences using a fish-eye lens camera that was set to the locomotion direction of the robot. The 235-deg FOV provided wide differences in the scene appearances depending on the locomotion direction, even at similar points. Figure 7 depicts sample images of appearance differences $\mathrm{CW}$ and $\mathrm{CCW}$ at a similar point. These images indicate that vision-based location recognition is a challenging task of computer vision (CV) studies.
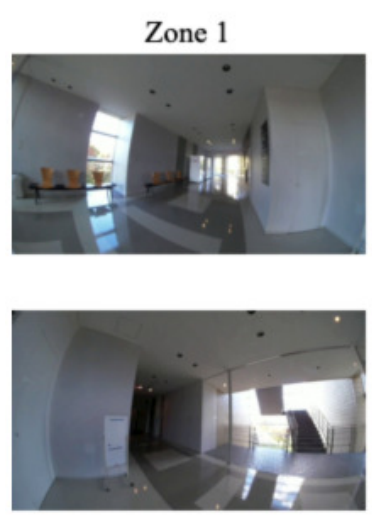
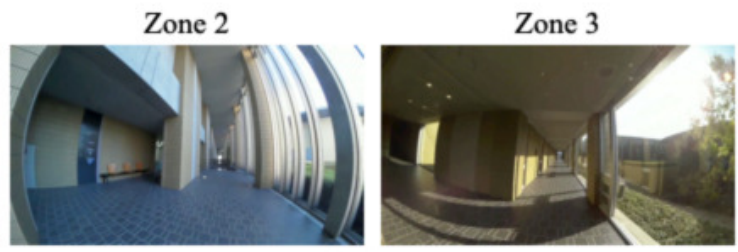

(a) $\mathrm{CW}$
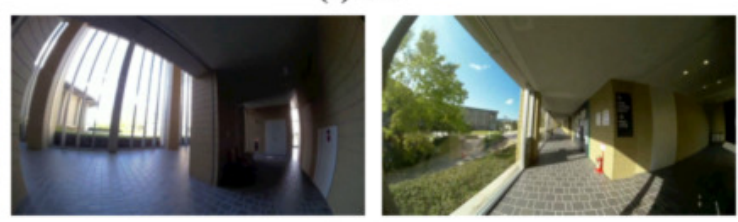

(b) $\mathrm{CCW}$
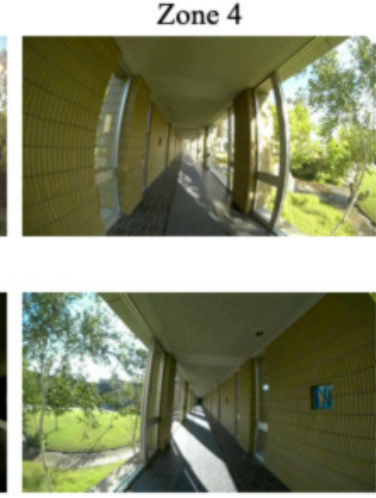

Figure 7. Scene appearance differences depending on locomotion directions at similar positions

Figure 8 depicts appearance differences of scene images at the same points in the three seasons. High-salience regions and extracted feature points are superimposed on the images with color curves and dots. Feature points on the SD images included various indoor objects in addition to lush trees and lawns outdoors. Moreover, feature points appeared in the buildings through transparent glass walls and windows. 

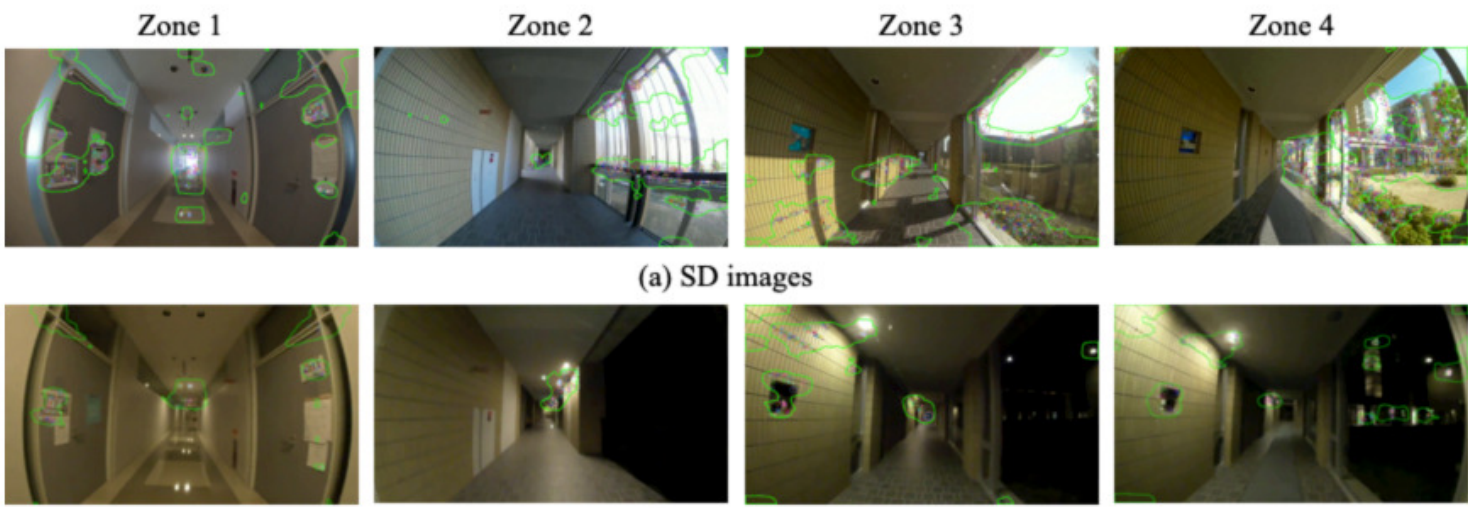

(b) AD images
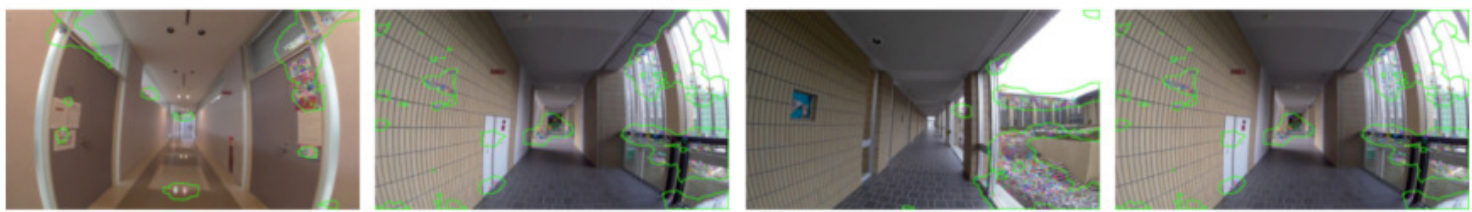

(c) WD images

Figure 8. Scene appearance differences depending on season at similar positions.

Feature points on $\mathrm{AD}$ images included indoor objects in addition to halation from indoor lighting reflected in transparent glass. Images without halation have no feature points. Compared with those of the SD images, the feature points are few. For WD images, the ground surface was covered slightly with snow. Although the sunlight was not intense, the indoor brightness was sufficient to extract object features for VL candidates. Numerous feature points were extracted both indoors and outdoors, similarly to the SD images.

\subsection{Extraction of Image Features}

Figure 9 depicts comparison results of extracted features. This example image includes a person as a moving object in the left panels. The middle panels depict saliency regions as VL candidates. The right panels depict extracted features from high saliency regions. The feature points are distributed over the person. Using YOLO, the human region is excluded from salient regions. The AKAZE features overlapped on the person disappeared.
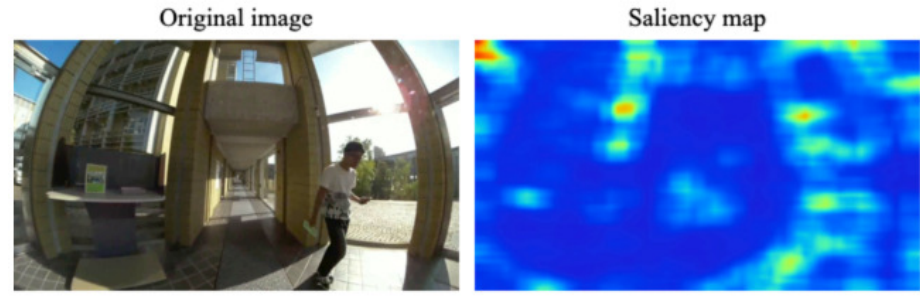

(a) Without YOLO
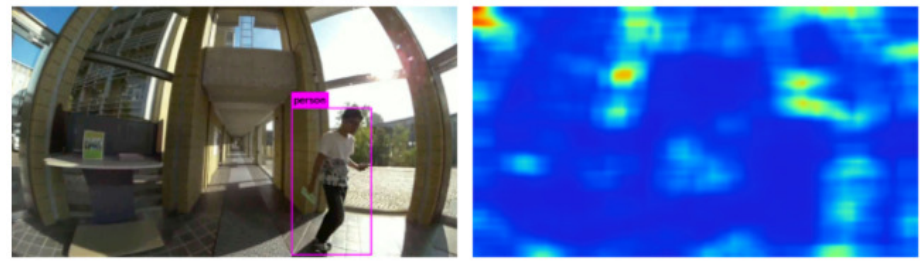

(b) With YOLO
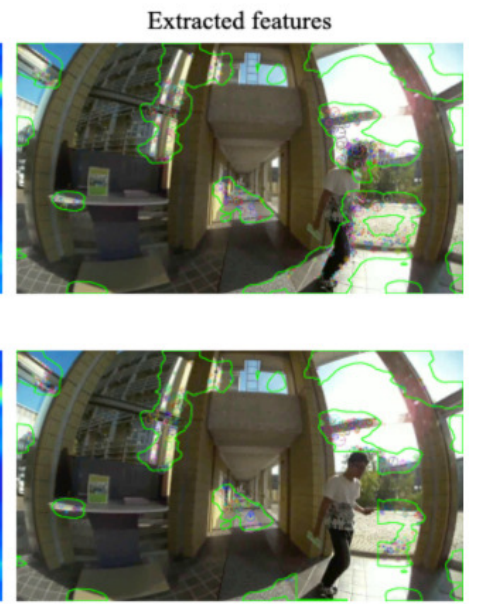

Figure 9. Results of comparison of extracted features with and without You Only Live Once (YOLO). 


\section{Evaluation Experiment}

\subsection{Benchmark and Evaluation Criteria}

We conducted an evaluation experiment to develop VL-based positional scene recognition from scene images obtained using a mobile robot. Table 3 presents details of 18 datasets in each season and the locomotion direction. We used leave-one-out cross-validation [60] as evaluation criteria. Specifically, a set of datasets in each season and direction was divided into two groups: one dataset was left for validation; the other datasets were used for training. We calculate the respective recognition accuracies for the five patterns of divided zones.

Table 3. Attributes of datasets [images].

\begin{tabular}{cccccccc}
\hline & \multicolumn{2}{c}{ First Round } & \multicolumn{2}{c}{ Second Round } & \multicolumn{2}{c}{ Third Round } \\
\hline Datasets & CW & CCW & CW & CCW & CW & CCW & Average \\
\hline SD & 2366 & 2350 & 2366 & 2356 & 2361 & 2354 & 2359 \\
AD & 2403 & 2346 & 2350 & 2357 & 2362 & 2352 & 2362 \\
WD & 2359 & 2371 & 2356 & 2355 & 2351 & 2344 & 2356 \\
\hline Average & 2376 & 2356 & 2357 & 2356 & 2358 & 2350 & 2359 \\
\hline
\end{tabular}

For evaluation criteria, the recognition accuracy $R_{a c c}[\%]$ for a validation dataset is defined as

$$
R_{\text {acc }}=\frac{N_{\text {correct }}}{N_{\text {total }}} \times 100
$$

where $N_{\text {total }}$ and $N_{\text {correct }}$ respectively represent the total numbers of validation images and correct recognition images that matched zone labels as GT.

\subsection{Optimization of Parameters}

Before the scene recognition evaluation experiment, we conducted a preliminary experiment to optimize meta-parameters for the regulation of our proposed method. The optimization subjects are the following four parameters: $S$, which is related to expression and granularity and expression of codebooks; $Q$, which is related to the resolution and expression of category maps; SOM learning iterations; and CPN learning iterations. Herein, the computational cost for the simultaneous optimization of these four parameters is an exponential multiple compared with a single case. Therefore, we optimized them sequentially. For this parameter optimization experiment, we merely used the SD images.

Figure 10 depicts the optimization result of the number of mapping layer units: $S$ for SOMs and $S$ for CPNs. We changed $S=2^{n}$ from $n=5$ to $n=10$ at $n=1$ intervals. The optimization experiment results revealed that $R_{a c c}$ improved steadily from $n=5$ to $n=7$. The local maximum of $52.3 \%$ was obtained at $n=7$, which corresponds to $Q=128$ units. The accuracies were reduced in other unit sizes. Therefore, the optimal size of the SOM mapping layer was ascertained as 128 units.

For $\mathrm{CPN}$, we changed $Q$ from $30 \times 30$ units to $80 \times 80$ units at $10 \times 10$ unit intervals. The optimization experiment results revealed that $R_{a c c}$ improved steadily from $30 \times 30$ units to $70 \times 70$ units. The local maximum of $51.1 \%$ was obtained at $70 \times 70$ units. Subsequently, $R_{a c c}$ dropped to $48.9 \%$ at $70 \times 70$ units. Therefore, the optimal size of the CPN mapping layer was ascertained as $70 \times 70$ units.

In addition, Figure 11 depicts the optimization result of learning iterations for SOMs and CPNs. Herein, $100 \times 10^{m}$ denotes learning iterations. We changed this parameter from $m=1$ to $m=6$ at $m=1$ intervals. The optimization experiment results of SOM learning iterations revealed that $R_{a c c}$ improved steadily according to the greater number of learning iterations. The maximum $R_{a c c}=52.9 \%$ was obtained at 10,000,000 iterations for SOMs. 


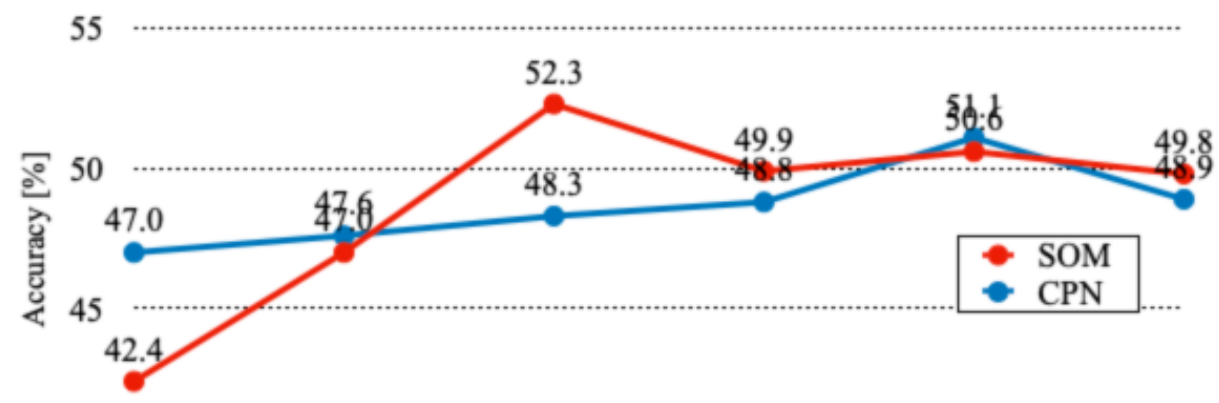

$\begin{array}{llllll}(32,30 \times 30) & (64,40 \times 40) & (128,50 \times 50) & (256,60 \times 60) & (512,70 \times 70) & (1024,80 \times 80)\end{array}$

(S, Q) [units]

Figure 10. Parameter experiment results of the number of self-organizing maps (SOMs) and counter propagation networks (CPNs) mapping units $(S, Q)$.

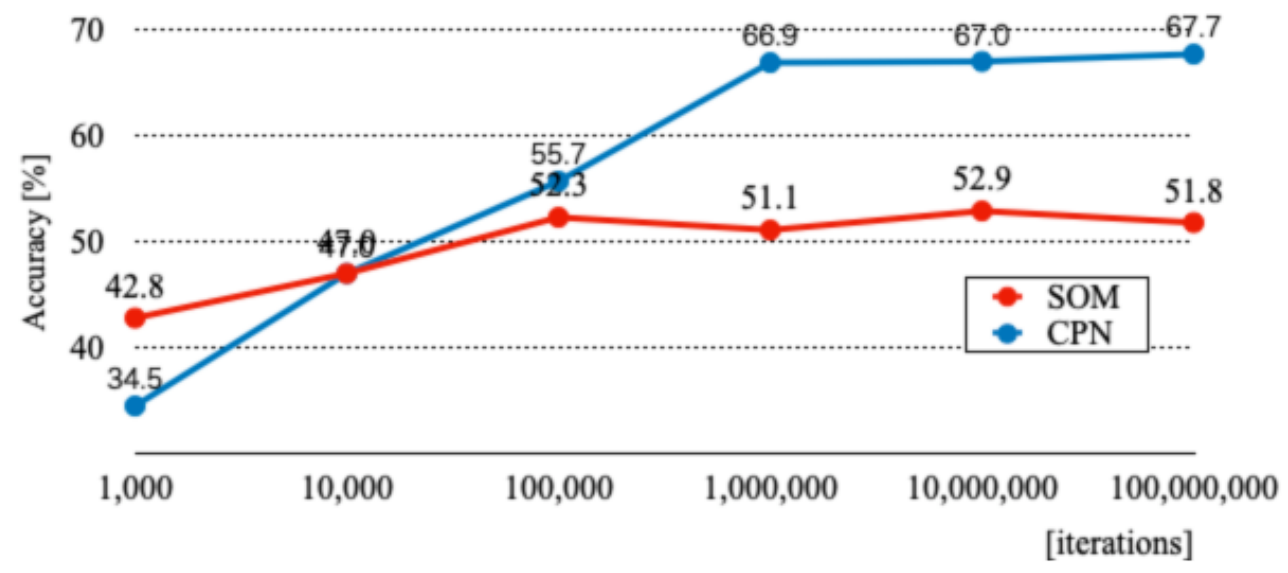

Figure 11. Parameter experiment results of learning iterations.

For CPN learning iterations, $R_{a c c}$ improved rapidly up to 1,000,000 iterations. Subsequently, $R_{a c c}$ improved gradually according to the greater number of learning iterations. Maximum $R_{a c c}=67.7 \%$ was obtained at $100,000,000$ iterations. However, the accuracy difference compared with $10,000,000$ iterations was only 0.7 percentage points. Moreover, the accuracy difference compared with $1,000,000$ iterations was a mere 0.8 percentage points. In addition to those slight differences achieved, the computation time increased exponentially. Therefore, this study showed the optimal CPN learning iterations at 1,000,000 iterations, given reasonable limitations of engineering, with balanced computational costs and accuracy.

\subsection{Positional Scene Recognition Results}

Figures 12-14 depict recognition accuracies for the respective datasets as shown in Table 3. As an overall tendency, the recognition accuracies vary depending on the season and the number of zones. The mean $R_{a c c}$ values of $\mathrm{SD}, \mathrm{AD}$, and WD were found, respectively, as $82.4 \%, 79.1 \%$, and $78.1 \%$. The experimentally obtained results revealed 4.3 percentage point difference in accuracy among the three datasets. 


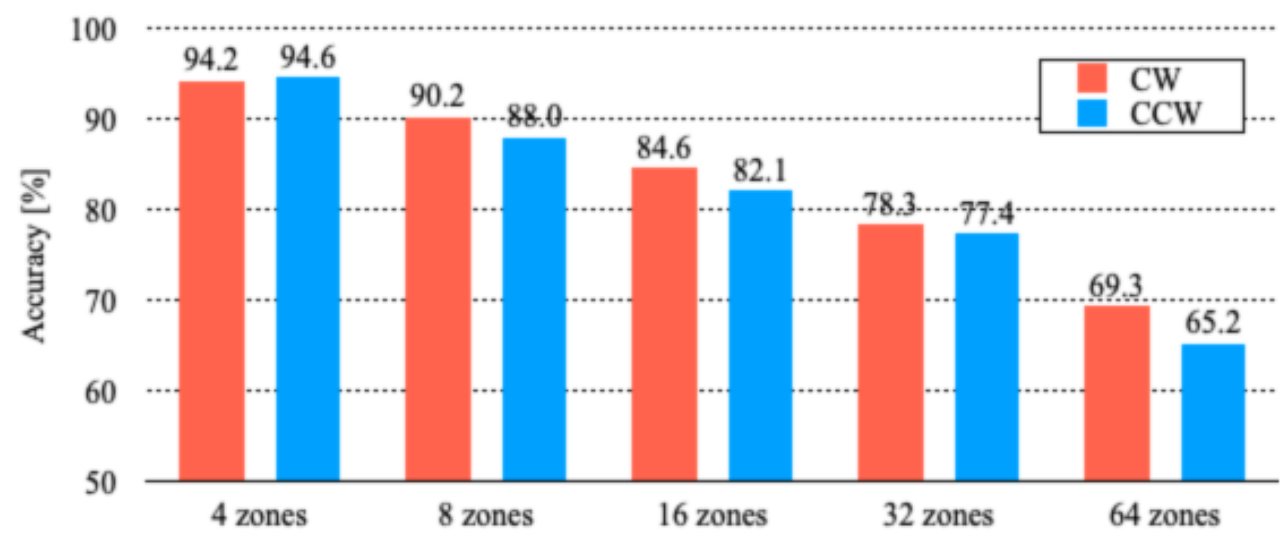

Figure 12. Recognition accuracy of summer datasets (SD).

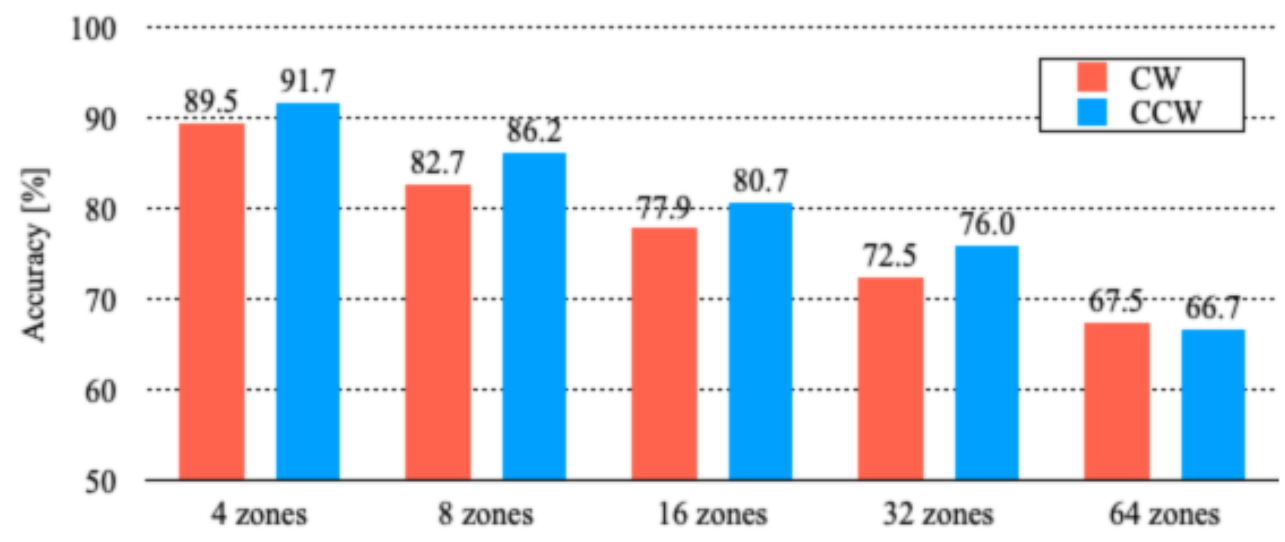

Figure 13. Recognition accuracy of autumn datasets (AD).

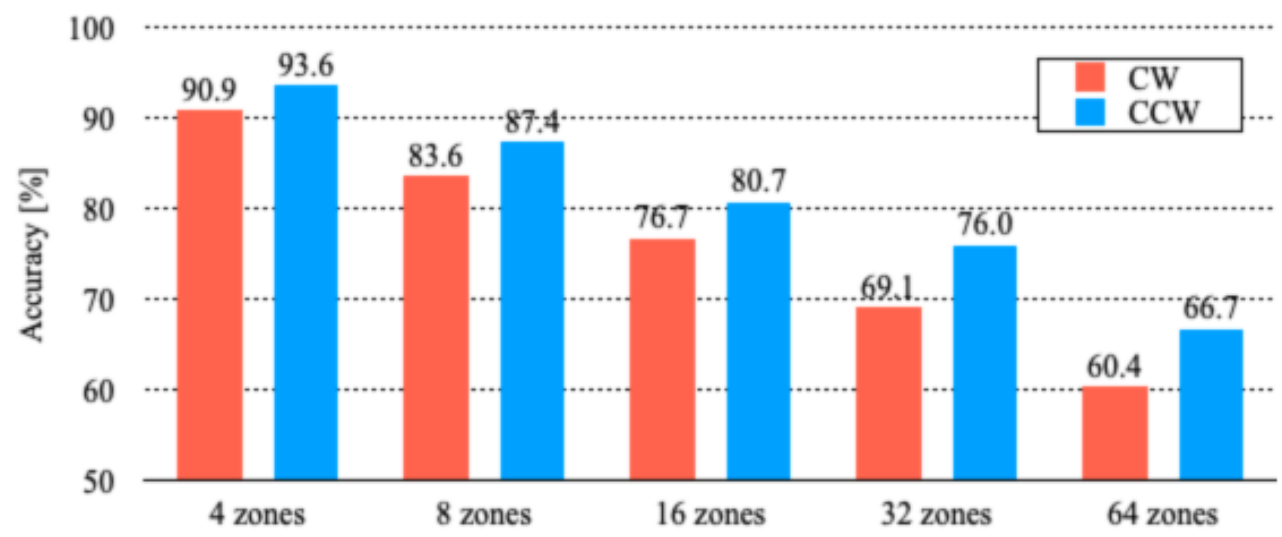

Figure 14. Recognition accuracy of winter datasets (WD).

For the locomotion direction for $\mathrm{SD}$, the mean $R_{a c c}$ of $\mathrm{CW}$ is 1.9 percentage points higher than that of CCW. For AD, the mean $R_{a c c}$ of CCW was 2.2 percentage points higher than that of CW. As a similar tendency to that of WD, the mean $R_{a c c}$ of $C C W$ was 4.7 percentage points higher than that of $C W$.

Recognition accuracy decreases according to a greater number of divided zones because of increasingly challenging levels for similar and overlapped images. Although $R_{a c c}$ of 64 zones of $\mathrm{CW}$ persists to the maximum of $69.3 \%$ for $\mathrm{SD}, R_{a c c}$ is maintained at a minimum of $60.4 \%$ for WD. Herein, a single-zone length for the case of 64 divisions with a total path length of $392 \mathrm{~m}$ is approximately $6 \mathrm{~m}$. When we subdivide the routes, we extract more information that is normally 
found in time series. However, our proposed method includes no mechanism to extract time-series features due to no recurrent structure. Therefore, the recognition accuracies were decreased according to the increased number of divided zones. Regarding seasonal changes, we assumed that AD images would have fewer outdoor features and improved recognition accuracy because of the fall of leaves on trees, as opposed to SD images. Similarly, we assumed that the WD images would be further improved by the snowfall. Although the overall recognition accuracy varies with the season, the experimentally obtained results revealed no contribute to the reduction of the decline as the number of zones increases. On the other hand, the results revealed that our proposed method provided $R_{a c c}$ of more than $60 \%$ with a resolution of $6 \mathrm{~m}$ for a monotonous indoor environment such as this corridor, which is greatly affected by the outdoors. That result was achieved solely using visual information obtained using a monocular camera with no GNSS or odometry.

\subsection{Analysis and Discussion}

We analyzed detailed recognition results in each zone using a confusion matrix. For this experiment, we visualized the whole tendency of recognition results obtained using a heatmap because the maximum division is 64 zones. Figure 15 depicts confusion matrixes using a heatmap. We designate it as a heatmap confusion matrix (HCM), which can check all zone results at a glance.
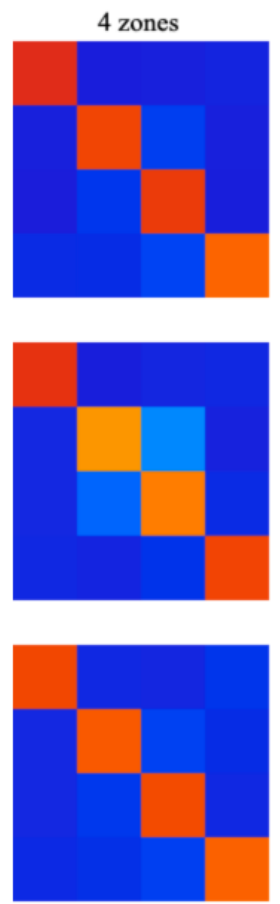

8 zones
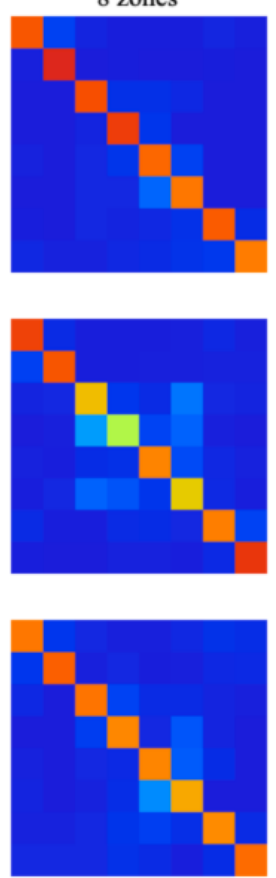

16 zones

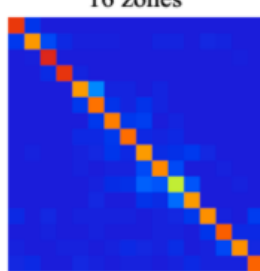

(a) SD

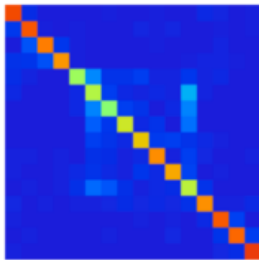

(b) $\mathrm{AD}$

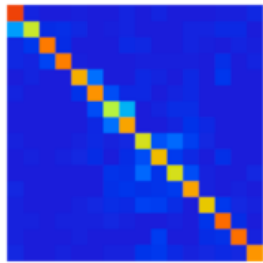

(c) WD
32 zones
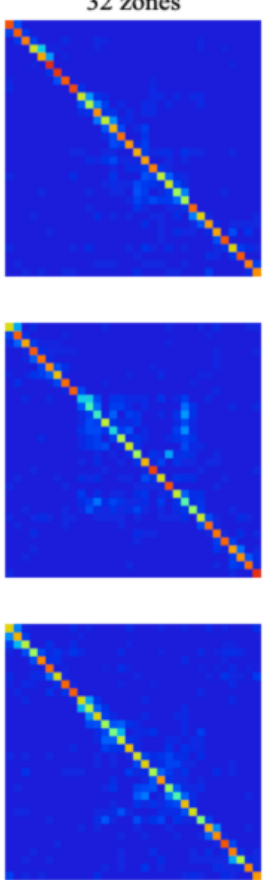

64 zones
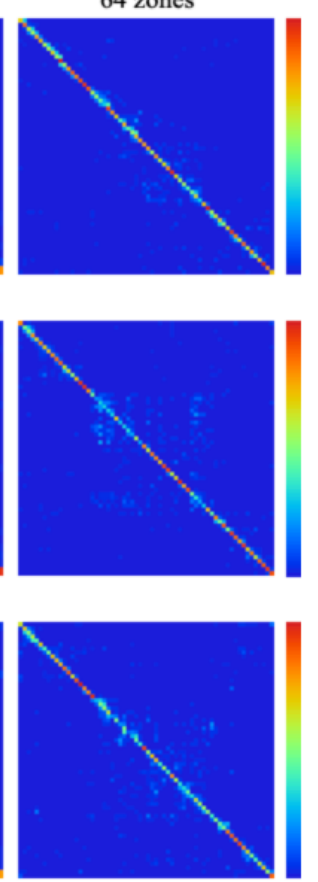

Figure 15. Heatmap confusion matrix.

In HCMs, correct results are distributed diagonally from the upper left to the lower right. A high color temperature result of this diagonal line represents high recognition accuracy. The distribution of higher color temperature distant from the diagonal line represents false recognition. Therefore, the HCM displays correct recognition as a high color temperature and false recognition as a low color temperature

For all HCMs, as depicted in Figure 15, we integrated CW and CCW results because we evaluated the difference of the locomotion direction, as depicted in Figures 12-14. The experimentally obtained HCMs revealed that a greater number of zone divisions corresponds to decreased diagonal color temperature. Nevertheless, no distribution of false recognition resembling hot spots, diffused false 
recognition appeared overall. In three datasets associated with different seasons, SD achieved the highest recognition accuracy.

Particularly, Figure 16 depicts the results of 16 zones in SD. For this result, Zones 1-4 and Zones 13-16 show high accuracy. In contrast, false recognition occurred in Zones 5-12. Particularly, numerous instances of false recognition occurred in Zones 6 and 12. The left panel of Figure 16 depicts images in Zones 1-4 with high accuracy. The path along which the robot moved in the corridor surrounded by lecture rooms has a small effect from outdoors. The right panel depicts images in Zones 9-12 with low accuracy. The scenes surrounded by transparent glass walls and windows of both sides exhibit a strong effect from outdoors.

As a benefit of our proposed method, the relation between zones can be visualized as a similarity in a low-dimensional space using a category map that is generated through learning based on competition and the neighborhood. Figure 17 depicts category maps created as learning results for the respective seasonal datasets. Each column of the figure depicts the category maps for which the number of divided routes is changed from 4 zones to 64 zones. The heatmap bars presented on the right side of each category map are divided according to the total number of divided zones. Clusters are created on a category map from similarities among categories. By contrast, complex features with inconsistent relations are distributed in multiple small clusters. The respective category maps demonstrated that clusters are distributed at multiple locations. This distribution property suggests the complexity of image features in each zone in VL-based semantic positional scene recognition.

For analysis of mapping properties of category maps, we used U-Matrix to extract cluster boundaries. Based on distances among weights on a category map, U-Matrix extracted similarity of neighbor units. We visualized boundary depths using a heatmap. Low similarity weights, which are also regarded as having low similarity between units, are displayed for lower color temperatures. In contrast, high similarity weights, which are considered to have high similarity among units, are displayed for higher color temperatures.
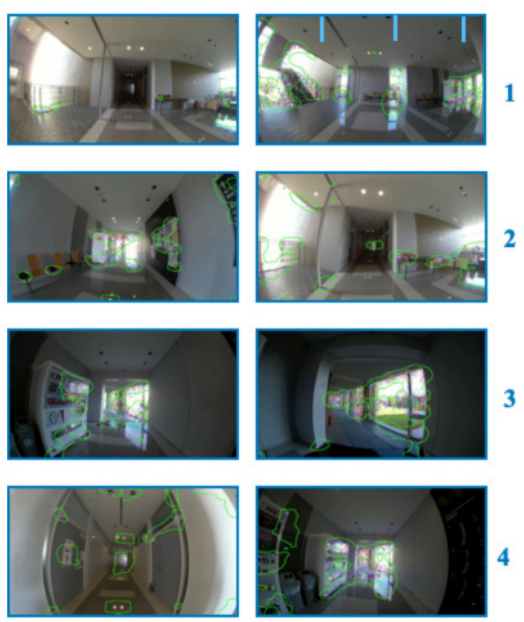

High accuracy zones

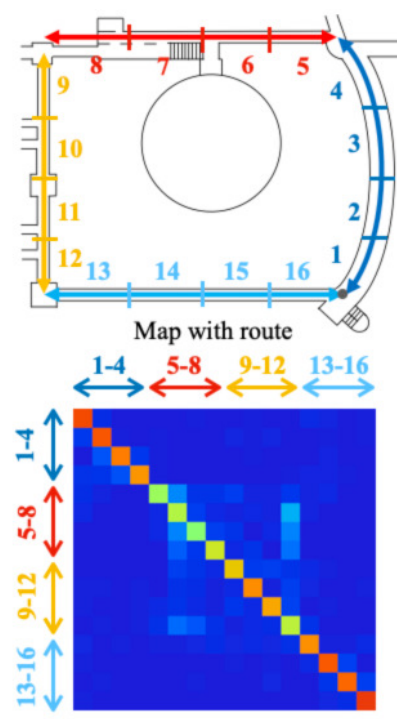

Confusion matrix
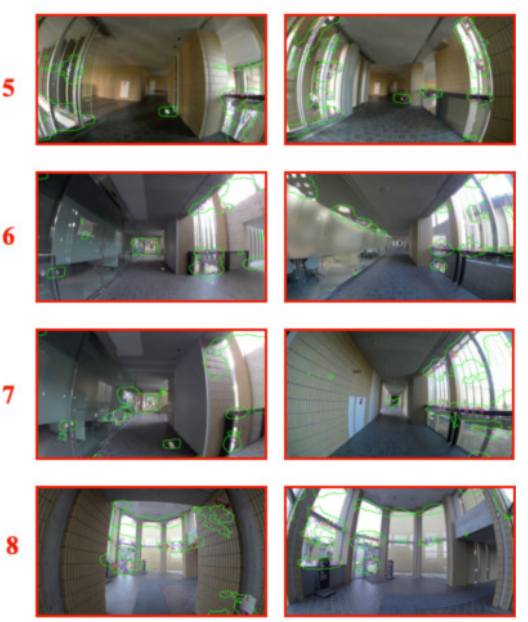

Low accuracy zones

Figure 16. Heatmap confusion matrix (HCM) example with 16 zones. 

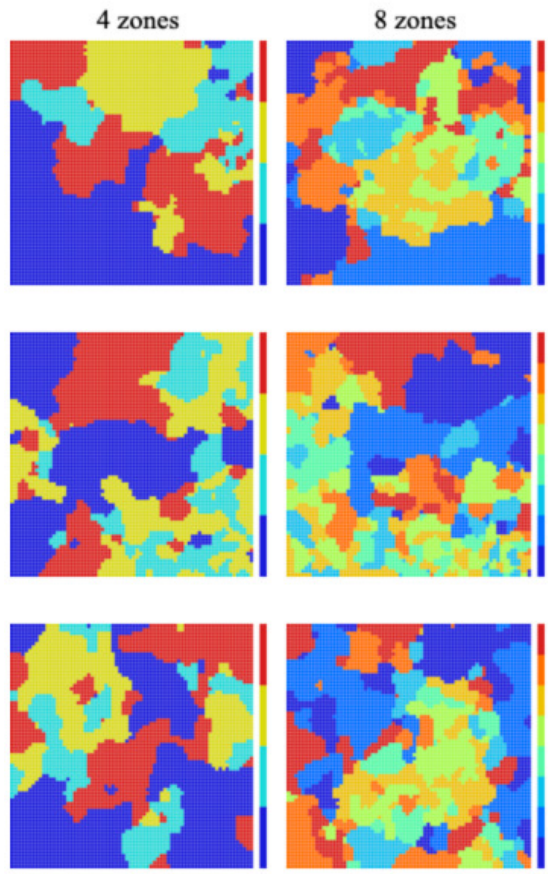

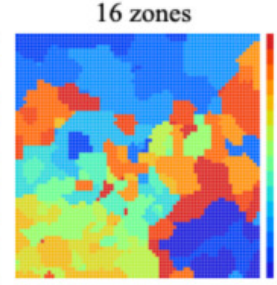

(a) $\mathrm{SD}$

32 zones

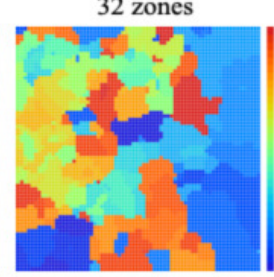

(c) WD

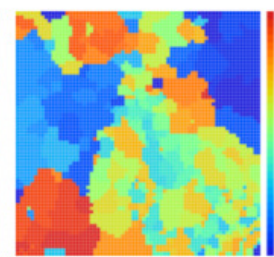

(b) $\mathrm{AD}$
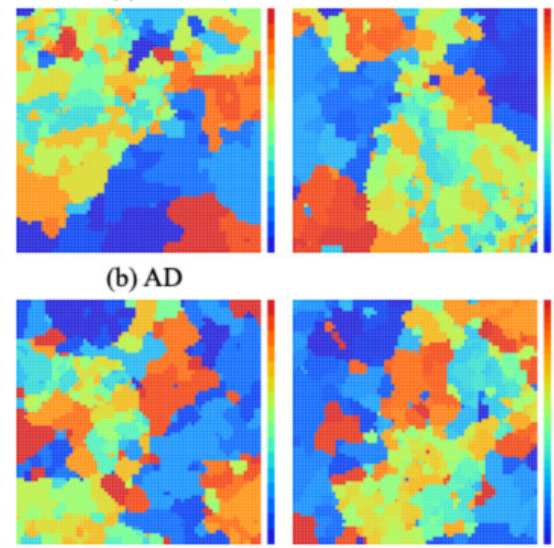

64 zones
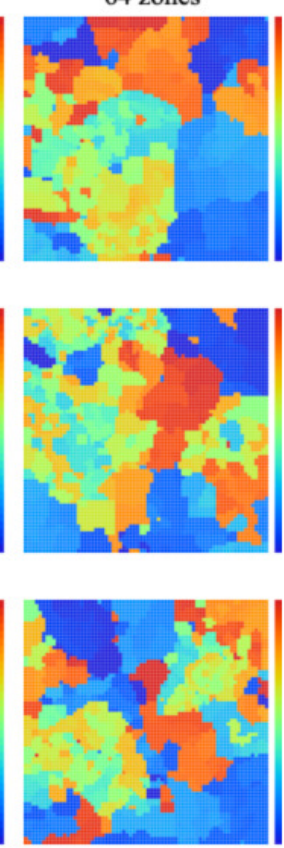

Figure 17. Results of category maps that visualize inter-zone relations.

Figure 18 depicts extraction results of cluster boundaries obtained using U-Matrix for all datasets of the respective divided zones. The white circles depict independent clusters that are surrounded by boundaries as high temperatures. Ideally, a suitable model comprises boundaries with low color temperatures inside clusters and high temperatures outside clusters. In this case, clear boundaries are obtained on a category map. Such a model is derived from highly independent feature distributions in a scene image. Nevertheless, scene appearances include similar and contradictory features for zones that are divided evenly along with the locomotion path, as depicted in Figure 6. Therefore, U-Matrix provided no distribution clusters according to the number of zones, similar to the results of category maps. The tendency obtained from this experimentally obtained result demonstrated that the clusters changed from a large cluster to a small cluster on U-Matrix for the number of zone increases. Our method handled the difficulty by which sets for dividing a scene are more complicated and difficult.

Rapidly progressed DL networks have been applied to various challenges posed by CV and RV. Our method, which used YOLO [39] to some degree, showed benefits from advanced DL performance. Dramatic improvement of recognition accuracy is expected to be sufficient using DL networks for the feature-extraction module for preprocessing and for the recognition module for recognition. Based on our earlier study [32], this study was aimed at developing original indoor benchmark datasets that include numerous transparent glass walls and windows to evaluate robustness for environmental changes outdoors. Our method was developed using classical ML-based algorithms without using DL because we visualized relations and similarities of scene images using category maps and U-Matrix for zone-based positional recognition with several granularity types.

For application to different purposes and benchmarks, we replaced the ML with a DL recognition module in our earlier study [41]. We then demonstrated the accuracy and computational cost differences between DL and ML. Actually, CV algorithms require both offline batch processing and online real-time processing. By contrast, RV algorithms fundamentally require online real-time processing from limited computational resources, especially for small robots including drones. Technological development and evolution related to cutting-edge AI devices [61] are expected to improve the shortcomings related to computational costs for DL algorithms. As a present optimal 
solution, we used ML algorithms with efficiency for current robots with no computational enhancement. The recognition module can be replaced with DL if accuracy is the highest priority for a target system. The input interface for this case can be set to the feature-extraction module but also directly to the recognition module. In consideration of such a replacement, we divided our proposed method into two independent modules.
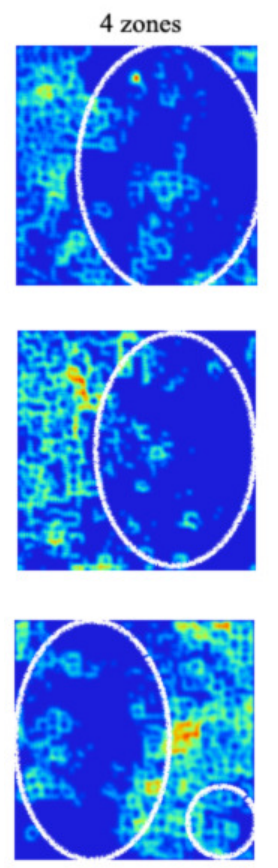

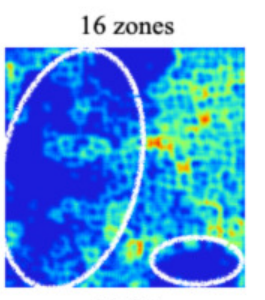

(a) SD
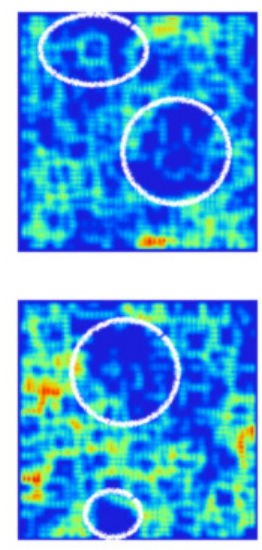

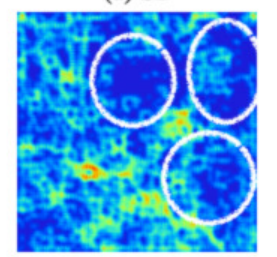

(b) $\mathrm{AD}$

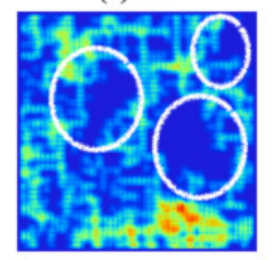

(c) WD
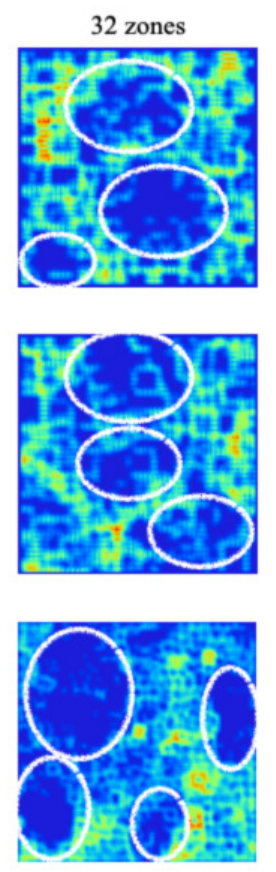
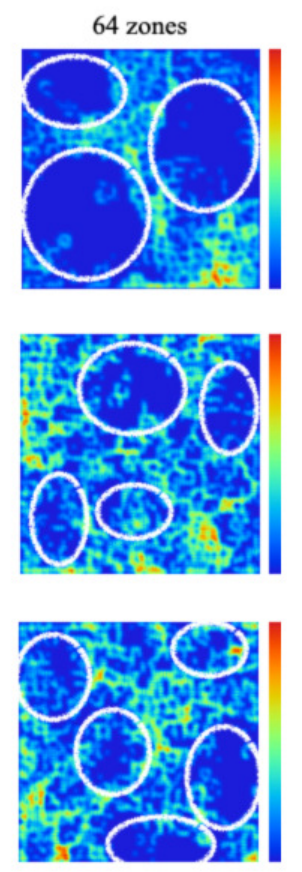

Figure 18. Results of U-Matrix extraction of cluster boundaries.

\section{Conclusions}

This paper presented a vision-based positional scene recognition method for an autonomous mobile robot using visual landmarks in an actual environment of coexisting humans and robots. We developed original benchmark datasets that include indoor-outdoor visual features in environments surrounded by transparent glass walls and windows. To include various and diverse changes of scene appearances, the datasets include two locomotion directions and three seasons. We conducted evaluation experiments for VL-based location recognition using datasets of time-series scene images obtained from a wide FOV camera mounted on a robot that ran a $392 \mathrm{~m}$ route that was divided into 64 zones with fixed intervals. We verified the performance and characteristics of meta-parameter optimization, mapping characteristics to category maps, and GT-based recognition accuracy. Moreover, we visualized similarity between scene images using category maps. We also visualized the relation of weights using U-Matrix.

As a subject of future work, we expect to introduce Elman-type feedback neural networks combined with deep learning mechanisms as a framework for learning time-series feature changes. We expect to extract flexible and variable zones based on visual changes obtained from category maps and U-Matrix. Moreover, we expect to implement our proposed method as online processing instead of the current offline processing for applying human-symbiotic robots of various types and various environments. Furthermore, we would like to open all the images as a novel open benchmark dataset after inserting semantic information including segmentation, recognition, labeling, GT, annotations, and security and privacy treatment. 
Author Contributions: Conceptualization, H.M. and K.S.; methodology, H.M.; software, H.M.; validation, H.W., S.N. and K.S.; formal analysis, H.M.; investigation, H.M.; resources, H.M.; data curation, H.M.; writing-original draft preparation, H.M.; writing-review and editing, H.M.; visualization, H.W.; supervision, S.N.; project administration, K.S.; funding acquisition, H.M. All authors have read and agreed to the published version of the manuscript.

Funding: This research was supported by Japan Society for the Promotion of Science (JSPS) KAKENHI Grant Number 17K00384.

Conflicts of Interest: The authors declare that they have no conflict of interest. The funders had no role in the design of the study, in the collection, analyses, or interpretation of data, in the writing of the manuscript, or in the decision to publish the results.

\section{Abbreviations}

The following abbreviations are used in this manuscript:

1D One-dimensional

2D Two-dimensional

3D Three-dimensional

AD Autumn datasets

AI Artificial intelligence

AKAZE Accelerated KAZE

AR Augmented reality

BB Bounding boxes

BRIEF Binary robust independent elementary features

CPN Counter propagation networks

$\mathrm{CV} \quad$ Computer vision

$\mathrm{CW} \quad$ Clockwise

CCW Counter-clockwise

DL Deep learning

GT Ground truth

FAST features from accelerated segment test

FED Fast explicit diffusion

FM Feature map

FOV Field of view

HCM Heatmap-colored confusion matrix

HOG Histograms of oriented gradient

RANSAC RANdom SAmple Consensus

MOT Multiple object tracking

ML Machine learning

ORB Oriented FAST and rotated BRIEF

RV Robot vision

SLAM Simultaneous localization and mapping

SD Summer datasets

SM Saliency map

SOM Self-organizing maps

SIFT Scale-invariant feature transform

VL Visual landmarks

WD Winter datasets

WTA Winner-take-all

\section{References}

1. Alsamhi, S.H.; Ma, O.; Ansari, M.S. Survey on Artificial Intelligence Based Techniques for Emerging Robotic Communication. Telecommun Syst. 2019, 72, 483-503. [CrossRef]

2. Vanzo, A.; Riccio, F.; Sharf, M.; Mirabella, V.; Catarci, T.; Nardi, D. Who is Willing to Help Robots? A User Study on Collaboration Attitude. Int. J. Soc. Robot. 2019, 12, 589-598. [CrossRef] 
3. Do, H.M.; Pham, M.; Sheng, W.; Yang, D.; Liu, M. RiSH: A Robot-Integrated Smart Home for Elderly Care. Robot. Auton. Syst. 2019, 101, 74-92. [CrossRef]

4. Talbot, B.; Lam, O.; Schulz, R.; Dayoub, F.; Upcroft, B.; Wyeth, G. Find My Office: Navigating Real Space From Semantic Descriptions. In Proceedings of the IEEE International Conference on Robotics and Automation, Stockholm, Sweden, 16-21 May 2016.

5. Khaliq, A.A.; Pecora, F.; Saffiotti, A. Children Playing with Robots Using Stigmergy on a Smart Floor. In Proceedings of the IEEE International Conferences on Ubiquitous Intelligence \& Computing, Advanced and Trusted Computing, Scalable Computing and Communications, Cloud and Big Data Computing, Internet of People, and Smart World Congress, Toulouse, France, 18-21 July 2016.

6. Shibata, T.; Kawaguchi, Y.; Wada, K. Investigation on People Living with Paro at Home. In Proceedings of the 19th International Symposium in Robot and Human Interactive Communication, Viareggio, Italy, 12-15 September 2010.

7. Kumar, B.; Sharma, L.; Wu, S. Job Allocation Schemes for Mobile Service Robots in Hospitals. In Proceedings of the IEEE International Conference on Bioinformatics and Biomedicine, Madrid, Spain, 3-6 December 2018.

8. Alatise, M.B.; Hancke, G.P.A Review on Challenges of Autonomous Mobile Robot and Sensor Fusion Methods. IEEE Access 2020, 8, 39830-39846. [CrossRef]

9. Koppula, H.S.; Saxena, A. Anticipating Human Activities Using Object Affordances for Reactive Robotic Response. IEEE Trans. Pattern Anal. Mach. Intell. 2016, 38, 14-29. [CrossRef]

10. Cadena, C.; Carlone, L.; Carrillo, H.; Latif, Y.; Scaramuzza, D.; Neira, J.; Reid, I.; Leonard, J.J. Past, Present, and Future of Simultaneous Localization and Mapping: Toward the Robust-Perception Age. IEEE Trans. Robot. 2016, 32, 1309-1332. [CrossRef]

11. Stumberg, L.; Usenko, V.; Engel, J.; Stúckler, J.; Cremers, D. From monocular SLAM to autonomous drone exploration. In Proceedings of the European Conference on Mobile Robots (ECMR), Paris, France, 6-8 September 2017.

12. Hayet, J.B.; Lerasle, F.; Devy, M. A Visual Landmark Framework for Indoor Mobile Robot Navigation. In Proceedings of the IEEE International Conference on Robotics and Automation, Washington, DC, USA, 11-15 May 2002.

13. Lazanas, A.; Latombe, J. Landmark-Based Robot Navigation. Algorithmica 1995, 13, 472-501. [CrossRef]

14. Mata, M.; Armingol, J.M.; Escalera, A.; Salichs, M.A. A visual landmark recognition system for topological navigation of mobile robots. In Proceedings of the IEEE International Conference on Robotics and Automation, Seoul, Korea, 21-26 May 2001.

15. Watman, C.; Austin, D.; Barnes, N.; Overett, G.; Thompson, S. Fast Sum of Absolute Differences Visual Landmark Detector. In Proceedings of the IEEE International Conference on Robotics and Automation, New Orleans, LA, USA, 26 April-1 May 2004.

16. Mohareri, O.; Rad, A.B. Autonomous humanoid robot navigation using augmented reality technique. In Proceedings of the IEEE International Conference on Mechatronics, Istanbul, Turkey, 13-15 April 2011.

17. Fischler, M.A.; Bolles, R.C. Random Sample Consensus: A Paradigm for Model Fitting with Applications to Image Analysis and Automated Cartography. Commun. ACM 1981, 24, 381-395. [CrossRef]

18. Ammirato, P.; Poirson, P.; Park, E.; Kośecká, J.; Berg, A.C. A Dataset for Developing and Benchmarking Active Vision. In Proceedings of the IEEE International Conference on Robotics and Automation, Marina Bay Sands, Singapore, 29 May-3 June 2017.

19. Firman, M. RGBD Datasets: Past, Present and Future. In Proceedings of the IEEE Conference on Computer Vision and Pattern Recognition Workshops, Las Vegas, NV, USA, 27-30 June 2016.

20. Georgakis, G.; Reza, M.A.; Mousavian, A.; Le, P.H.; Kosecka, J. Multiview RGB-D Dataset for Object Instance Detection. In Proceedings of the Fourth International Conference on 3DVision, Stanford, CA, USA, 25-28 October 2016.

21. Song, S.; Lichtenberg, S.; Xiao, J. Sun RGB-D: A RGB-D Scene Understanding Benchmark Suite. In Proceedings of the 28th IEEE Conference on Computer Vision and Pattern Recognition, Boston, MA, USA, 7-12 June 2015.

22. Singh, A.; Sha, J.; Narayan, K.S.; Achim, T.; Abbeel, P. Bigbird: A Large-Scale 3D Database of Object Instances. In Proceedings of the IEEE International Conference on Robotics and Automation, Hong Kong, China, 31 May-7 June 2014.

23. Lai, K.; Bo, L.; Ren, X.; Fox, D. A Large-Scale Hierarchical Multi-View RGB-D Object Dataset. In Proceedings of the IEEE International Conference on Robotics and Automation, Shanghai, China, 9-13 May 2011. 
24. Wen, L.; Zhu, P.; Du, D.; Bian, X.; Ling, H.; Hu, Q.; Zheng, J.; Peng, T.; Wang, X.; Zhang, Y.; et al. VisDrone-MOT2019: The Vision Meets Drone Multiple Object Tracking Challenge Results. In Proceedings of the IEEE/CVF International Conference on Computer Vision Workshop, Seoul, Korea, 27-28 October 2019. [CrossRef]

25. Huang, Y.; Sithole, L.; Lee, T. Structure from Motion Technique for Scene Detection Using Autonomous Drone Navigation. IEEE Trans. Syst. Man, Cybern. Syst. 2019, 49, 2559-2570. [CrossRef]

26. Madokoro, H.; Sato, K.; Shimoi, N. Vision-Based Indoor Scene Recognition from Time-Series Aerial Images Obtained Using a MAV Mounted Monocular Camera. Drones 2019, 3, 22. [CrossRef]

27. Vetrella, A.R.; Fasano, G.; Accardo, D.; Moccia, A. Differential GNSS and Vision-Based Tracking to Improve Navigation Performance in Cooperative Multi-UAV Systems. Sensors 2016, 16, 2164. [CrossRef] [PubMed]

28. Quattoni, A.; Torralba, A. Recognizing indoor scenes. In Proceedings of the IEEE Conference on Computer Vision and Pattern Recognition, Miami, FL, USA, 20-25 June 2009.

29. Raja, R.; Roomi, S.M.; Dharmalakshmi, D. Outdoor scene classification using invariant features. In Proceedings of the Fourth National Conference on Computer Vision, Pattern Recognition, Image Processing and Graphics, Jodhpur, India, 18-21 December 2013.

30. Tahir, W.; Majeed, A.; Rehman, T. Indoor/outdoor image classification using GIST image features and neural network classifiers. In Proceedings of the 12th International Conference on High-Capacity Optical Networks and Enabling/Emerging Technologies, Islamabad, Pakistan, 21-23 December 2015; pp. 1-5.

31. Gilroy, S.; Jones, E.; Glavin, M. Overcoming Occlusion in the Automotive Environment-A Review. IEEE Trans. Intell. Transp. Syst. 2019, 1-13.

32. Madokoro, H.; Sato, K.; Shimoi, N. Indoor Scene and Position Recognition Based on Visual Landmarks Obtained from Visual Saliency without Human Effect. Robotics 2019, 8, 3. [CrossRef]

33. Itti, L.; Koch, C.; Niebur, E. A Model of Saliency-Based Visual Attention for Rapid Scene Analysis. IEEE Trans. Pattern Anal. Mach. Intell. 1998, 20, 1254-1259. [CrossRef]

34. Alcantarilla, P.F.; Nuevo, J.; Batoli, A. Fast Explicit Diffusion for Accelerated Features in Nonlinear Scale Spaces. In Proceedings of the British Machine Vision Conference, Bristol, UK, 9-13 September 2013.

35. Dalal, N.; Triggs, B. Histograms of oriented gradients for human detection. In Proceedings of the IEEE Computer Society Conference on Computer Vision and Pattern Recognition, San Diego, CA, USA, 20-25 June 2005.

36. Kohonen, T. Self-Organized formation of Topologically Correct Feature Maps. Biol. Cybern. 1982, 43, 59-69. [CrossRef]

37. Nielsen, R.H. Counterpropagation networks. Appl. Opt. 1987, 26, 4979-4983. [CrossRef]

38. Fukushi, R.; Madokoro, H.; Sato, K. Semantic Scene Recognition and Zone Labeling for Mobile Robot Benchmark Datasets based on Category Maps. In Proceedings of the 18th International Conference on Control, Automation and Systems, Daegwallyeong, Korea, 17-20 October 2018; pp. 475-480.

39. Redmon, J.; Divvala, S.; Girshick, R.; Farhadi, A. You Only Look Once: Unified, Real-Time Object Detection. In Proceedings of the IEEE Conference on Computer Vision and Pattern Recognition, Las Vegas, NV, USA, 27-30 June 2016.

40. Ultsch, A. Clustering with SOM U*C. In Proceedings of the Workshop on Self-Organizing Maps, Paris, France, 5-8 September 2005.

41. Madokoro, H.; Woo, H.; Sato, K. Comparative Evaluation of Input Features Used for Deep Neural Networks to Recognize Semantic Indoor Scene from Time-Series Images Obtained Using Mobile Robot. In Proceedings of the Fourteenth International Conference on Software Engineering Advances, Valencia, Spain, 24-28 November 2019.

42. Marrand, D.; Poggio, T. A computational theory of human stereo vision. Proc. R. Soc. B 1979, 204, 301-328.

43. Snyder, A.W.; Laughlin, S.B.; Stavenga, D.G. Information capacity of eyes. Vis. Res. 1977, 17, $1163-1175$. [CrossRef]

44. Lowe, D.G. Object Recognition from Local Scale-Invariant Features. In Proceedings of the IEEE International Conference Computer Vision, Corfu, Greece, 20-25 September 1999.

45. Bay, H.; Ess, A.; Tuytelaars, T.; Gool, L.V. SURF: Speeded up robust features. Comput. Vis. Image Underst. 2008, 110, 346-359. [CrossRef]

46. Calonder, M.; Lepetit, V.; Strecha, C.; Fua, P. BRIEF: Binary Robust Independent Elementary Features. In Proceedings of the 11th European Conference on Computer Vision, Crete, Greece, 5-11 September 2010. 
47. Rublee, E.; Rabaud, V.; Konolige, K.; Bradski, G. ORB: An Efficient Alternative to SIFT or SURF. In Proceedings of the International Conference on Computer Vision, Barcelona, Spain, 6-13 November 2011; pp. 2564-2571.

48. Alcantarilla, P.F.; Bartoli, A.; Davison, A.J. KAZE Features. In Proceedings of the 12th European Conference on Computer Vision, Florence, Italy, 7-13 October 2012.

49. Grewenig, S.; Weickert, J.; Bruhn, A. From box filtering to fast explicit diffusion. In Proceedings of the German Association for Pattern Recognition Symposium on Pattern Recognition, Darmstadt, Germany, 22-24 September 2010.

50. Weickert, J.; Romeny, B.H.; Viergever, M.A. Efficient and reliable schemes for nonlinear diffusion filtering. IEEE Trans. Image Process. 1988, 7, 398-410. [CrossRef]

51. Yang, X.; Cheng, K.T. LDB: An ultra-fast feature for scalable augmented reality. In Proceedings of the IEEE and ACM International Symposium on Mixed and Augmented Reality, Atlanta, GA, USA, 5-8 November 2012.

52. LeCun, Y.; Bengio, Y.; Hinton, G. Deep learning. Nature 2015, 521, 436-444. [CrossRef] [PubMed]

53. Ju, M.; Luo, H.; Wang, Z.; Hui, B.; Chang, Z. The Application of Improved YOLO V3 in Multi-Scale Target Detection. Appl. Sci. 2019, 9, 3775. [CrossRef]

54. Lan, W.; Dang, J.; Wang, Y.; Wang, S. Pedestrian Detection Based on YOLO Network Model. In Proceedings of the IEEE International Conference on Mechatronics and Automation, Changchun, China, 5-8 August 2018.

55. Benjdira, B.; Khursheed, T.; Koubaa, A.; Ammar, A.; Ouni, K. Car Detection using Unmanned Aerial Vehicles: Comparison between Faster R-CNN and YOLOv3. In Proceedings of the First International Conference on Unmanned Vehicle Systems_-Oman, Muscat, Oman, 5-7 February 2019.

56. Chen, R.C. Automatic License Plate Recognition via sliding-window darknet-YOLO deep learning. Image Vis. Comput. 2019, 87, 47-56.

57. Tian, D.; Zhang, C.; Duan, X.; Wang, X. An Automatic Car Accident Detection Method Based on Cooperative Vehicle Infrastructure Systems. IEEE Access 2019, 7, 127453-127463. [CrossRef]

58. Liu, W.; Anguelov, D.; Erhan, D.; Szegedy, C.; Reed, S.; Fu, C.Y.; Berg, A.C. SSD: Single Shot MultiBox Detector. Available online: https:/ / arxiv.org/abs/1512.02325 (accessed on 15 April 2020).

59. Cao, Z.; Hidalgo, G.; Simon, T.; Wei, S.E.; Sheikh, Y. OpenPose: Realtime Multi-Person 2D Pose Estimation using Part Affinity Fields. arXiv 2020, arXiv:1812.08008.

60. Kohavi, R. A study of cross-validation and bootstrap for accuracy estimation and model selection. In Proceedings of the Fourteenth International Joint Conference on Artificial Intelligence, Montreal, QC, Canada, 20-25 August 1995.

61. Wang, X.; Han, Y.; Wang, C.; Zhao, Q.; Chen X.; Chen, M. In-Edge AI: Intelligentizing Mobile Edge Computing, Caching and Communication by Federated Learning IEEE Netw. 2019, 33, 156-165. [CrossRef]

(C) 2020 by the authors. Licensee MDPI, Basel, Switzerland. This article is an open access article distributed under the terms and conditions of the Creative Commons Attribution (CC BY) license (http://creativecommons.org/licenses/by/4.0/). 